Simulating Time-Dependent Energy Transfer Between Crossed Laser Beams in an Expanding Plasma

J. A. F. Hittinger, M. R. Dorr, R. L. Berger, E. A. Williams

October 22, 2004

Journal of Computational Physics 
This document was prepared as an account of work sponsored by an agency of the United States Government. Neither the United States Government nor the University of California nor any of their employees, makes any warranty, express or implied, or assumes any legal liability or responsibility for the accuracy, completeness, or usefulness of any information, apparatus, product, or process disclosed, or represents that its use would not infringe privately owned rights. Reference herein to any specific commercial product, process, or service by trade name, trademark, manufacturer, or otherwise, does not necessarily constitute or imply its endorsement, recommendation, or favoring by the United States Government or the University of California. The views and opinions of authors expressed herein do not necessarily state or reflect those of the United States Government or the University of California, and shall not be used for advertising or product endorsement purposes. 


\title{
Simulating Time-Dependent Energy Transfer Between Crossed Laser Beams in an Expanding Plasma *
}

\author{
J. A. F. Hittinger*, M. R. Dorr \\ Center for Applied Scientific Computing, L-561 \\ Lawrence Livermore National Laboratory, \\ P.O. Box 808, Livermore, CA, 94551-0808 USA \\ R. L. Berger, E. A. Williams \\ AX Division, L-038 \\ Lawrence Livermore National Laboratory, \\ P.O. Box 808, Livermore, CA, 94551-0808 USA
}

\begin{abstract}
A coupled mode system is derived to investigate a three-wave parametric instability leading to energy transfer between co-propagating laser beams crossing in a plasma flow. The model includes beams of finite width refracting in a prescribed transverse plasma flow with spatial and temporal gradients in velocity and density. The resulting paraxial light equations are discretized spatially with a Crank-Nicholson-type scheme, and these algebraic constraints are nonlinearly coupled with ordinary differential equations in time that describe the ion acoustic response. The entire nonlinear differential-algebraic system is solved using an adaptive, backward-differencing method coupled with Newton's method. A numerical study is conducted in two di-
\end{abstract}


mensions that compares the intensity gain of the fully time-dependent coupled mode system with the gain computed under the further assumption of a strongly-damped ion acoustic response. The results demonstrate a time-dependent gain suppression when the beam diameter is commensurate with the velocity gradient scale length. The gain suppression is shown to depend on time-dependent beam refraction and is interpreted as a time-dependent frequency shift.

Key words: laser plasma interaction, forward Brillouin scattering, numerical algorithms, paraxial wave equation, coupled mode equations, differential-algebraic systems

1991 MSC: 65M06, 65L80, 77A99, 78A45

\section{Introduction}

[Fig. 1 about here.]

Laser-plasma interactions that lead to a resonant transfer of energy between crossed laser beams could affect indirect-drive inertial confinement fusion experiments to be performed at the National Ignition Facility (NIF) [1]. In such experiments, dozens of beams will cross in the plasma flowing supersonically from the hohlraum laser entrance hole (LEH). Inter-beam energy transfer

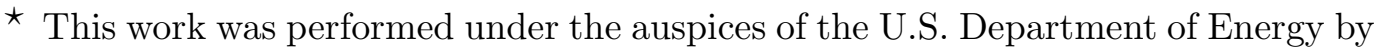
University of California, Lawrence Livermore National Laboratory under Contract W-7405-Eng-48. UCRL-JRNL-207422.

* Corresponding author.

Email addresses: hittinger1@llnl.gov (J. A. F. Hittinger), dorr1@llnl.gov

(M. R. Dorr), berger5@llnl.gov (R. L. Berger), williams16@llnl.gov (E. A. Williams). 
could redistribute the illumination within the hohlraum, adversely affecting the implosion symmetry required to achieve fusion.

The seminal work on inter-beam power transfer concerned beams of different frequencies crossing at a small angle in a stationary homogeneous plasma [2]. The transfer takes place through the well-known stimulated Brillouin scattering process wherein the ponderomotive force of the two laser beams resonantly drives an ion acoustic wave at the difference frequency and the wavevector of the two laser beams. Such a transfer process was demonstrated experimentally by comparing the power transmitted by a lower-frequency "probe" laser beam in the presence of a higher-frequency "pump" laser beam to the power transmitted either when the pump beam was absent or when the beam frequency difference failed to satisfy the forward Brillouin resonance condition of the transfer $[3,4]$.

Plasma flow can both detune the resonance when the laser beams have different frequencies or allow the interaction to occur when the beams have equal frequencies. When a plasma flow exists in which the velocity component in the ion acoustic wave direction is the plasma sound speed, but of opposite sign, the driven ion acoustic wave becomes a standing wave in the lab frame. In such a configuration, the stationary density grating imposed by the ion acoustic wave is wavenumber-matched to Bragg (Brillouin) scatter energy from the upstream pump beam to the downstream probe beam.

The power transfer in flowing plasma of equal frequency laser beams was shown in a series of experiments $[5,6]$. These experiments stimulated multidimensional simulations and theory that considered a variety of physical effects that influence the power transfer such as self-focusing and geometry [7], 
beam intensity non-uniformity and beam deflection of overlapping beams [8], nonlinear power transfer between different frequency beams in a homogeneous plasma in two and three dimensions $[9,10]$, and power transfer between beams that each have multiple frequencies [11] such as occurs with some beam smoothing techniques [12].

To understand better the potential impact of cross-beam energy transfer, a new series of experiments were performed at the University of Rochester's Laboratory for Laser Energetics [13]. In these experiments, heater beams exploded a foil, generating an expanding column of plasma. A pair of laser beams were crossed in this flow where the local plasma state nears the sonic resonance condition. The transmitted power in the pump and probe beams were measured and adjusted to compensate for inverse Bremsstrahlung absorption.

Although these experiments demonstrated power transfer that verified the underlying physics, the transfer was significantly less than that suggested by the simulations and the theory with a linear response of the ion wave to the ponderomotive drive. The amplification of the beam gaining power decreased as its initial amplitude increased, suggesting a nonlinear process. Simulations with the particle-in-cell (PIC) code, Zohar [14], in one dimension have shown that the ion waves are indeed driven to nonlinear levels which reduces the effectiveness of the power transfer because of kinetic effects such as nonlinear frequency shifts [15]. Recently, a PIC-inspired model for nonlinear frequency shifts has been used successfully to simulate the experiments provided the interaction length is limited to less than the full interaction length [16].

However, none of the theory and simulation efforts to date incorporate all the effects known to influence the experimental results. For example, in the exper- 
iments with plasma flow, the flow conditions satisfy the resonance condition for forward SBS for only a fraction of the beam cross section because of flow gradients across the beams and for only a limited duration because the flow evolution. In addition, the flow gradients are accompanied by electron density gradients which refract each beam and dynamically move the resonance surface.

In conjunction with the experimental work at Rochester, a numerical study was conducted using the Adaptive Laser Plasma Simulator (ALPS) [17]. In the ALPS code, a paraxial light model is coupled to a nonlinear, quasineutral fluid representation of the plasma. This model is capable of representing the hydrodynamic-scale velocity and density gradients and finite-beam interaction effects, but not kinetic nonlinearity of the ion waves. The numerical experiments initialized the plasma conditions with a self-similar rarefaction fan with a stationary sonic line. Unfortunately, the numerical solutions were unable to replicate the nonlinear saturation seen in the exploding-foil experiments - a result consistent with the hypothesis that the nonlinear saturation mechanism is a kinetic effect not modeled in the fluid equations. However, the numerical results regularly demonstrated an unexpected transient suppression of the energy transfer for plasma flows with large initial gradients relative to the beam diameters.

To further investigate this effect and to reduce the computational expense, we have developed the numerical model described in this paper, which demonstrates the same transient gain suppression. The mathematical model is a coupled-mode (3-wave) system similar to those used to study other plasma parametric instabilities, but specialized to this particular problem. We consider the interaction of pump and probe laser beams crossing in a quasineu- 
tral plasma freely expanding into a vacuum as shown in Figure 1. We assume that the beams have the same frequency and diameter and that the beams co-propagate with a small angle between them. This latter constraint allows for the use of a time-enveloped, paraxial description of the evolution of the complex light wave amplitudes similar to that used in the ALPS code. The plasma flow is modeled as a time-dependent, one-dimensional linearization about a self-similar, continuum rarefaction flow. We assume a harmonic form for the ion acoustic perturbations that nominally satisfy the three-wave resonant condition,

$$
\omega_{0}-\omega_{1}=0 \approx \pm\left|\mathbf{k}_{0}-\mathbf{k}_{1}\right| c_{s}+\left(\mathbf{k}_{0}-\mathbf{k}_{1}\right) \cdot \mathbf{v}
$$

where $\omega_{0}$ and $\omega_{1}$ are the pump and probe frequencies, respectively; $\mathbf{k}_{0}$ and $\mathbf{k}_{1}$ are the pump and probe wavenumber vectors respectively; $c_{s}$ is the ion acoustic speed; and $\mathbf{v}$ is the flow velocity. The discretized coupled-mode system is integrated in time using a differential algebraic system solver.

The assumption of a self-similar expansion allows the plasma flow to be characterized by a single parameter, $L_{\perp}$, the transverse velocity scale length. When the ion acoustic perturbations have a length scale much smaller than $L_{\perp}$, and in the limit of strong acoustic damping, the acoustic system reduces to an algebraic equation for the complex density perturbation amplitude in terms of the enveloped laser field amplitudes; this is a modest extension of the Rosenbluth gain formula [18]. At the other extreme, for problems in which the background scale length $L_{\perp}$ approaches the beam width or less, we will show that the motion of the beams due to their refraction in the evolving density profile introduces a temporal phase shift that modifies the resonant condition (1). The fact that time-dependence in background scattering media can affect the gain 
rate for parametric instabilities was demonstrated in $[19,20]$, in the latter case for backward stimulated Brillouin scattering in a one-dimensional, isothermal rarefaction. The analogous result presented here provides a quantitative description of this effect for the crossed beam scenario, and we have chosen to focus on this phenomenon in this paper instead of other gain modifying effects such as pump depletion or particle trapping.

In Section 2, we present the coupled-mode model. After some simplifications, a formula for the steady-state probe gain is presented. The numerical methods used to integrate the full, coupled mode system are described in Section 3. In Section 4, we present numerical results obtained using the model, with a final discussion in Section 5.

\section{Theoretical Model}

Consider the standard Euler-Poisson fluid model for a quasineutral plasma. For electron and ion number densities $n_{e}$ and $n_{i}$, respectively, the Poisson equation for the electrostatic potential $\phi$ is

$$
\epsilon_{0} \nabla^{2} \phi=e\left(n_{e}-Z n_{i}\right) \approx 0
$$

where $\epsilon_{0}$ is the permittivity of free space, $e$ is the fundamental charge, and $Z$ is the ionization state. Quasineutrality $\left(n_{e} \approx Z n_{i}\right)$ is a suitable assumption for length scales much larger than the Debye length, and this approximation allows one to describe the plasma dynamics using the fluid equations for a single agglomerated species.

We denote by $n \equiv n_{i} \approx n_{e} / Z$ the number density; $\mathbf{v}$ the velocity vector; $p$ 
the pressure; $m$ the composite mass per particle; and $\mathbf{f}_{p}(n, \mathcal{E})$ the specific ponderomotive force due to the laser electric field $\mathcal{E}$. Furthermore, we allow for the effects of a damping force $\mathbf{d}(\mathbf{v})$ on the plasma; the exact form for this damping will remain unspecified momentarily. Conservation of mass and momentum are thus expressed by

$$
\begin{aligned}
\partial_{t} n+(\mathbf{v} \cdot \nabla) n+n \nabla \cdot \mathbf{v} & =0 \\
\partial_{t} \mathbf{v}+(\mathbf{v} \cdot \nabla) \mathbf{v}+\frac{1}{m n} \nabla p & =\mathbf{f}+\mathbf{d}
\end{aligned}
$$

For an equation of state, we assume an ideal gas in local thermodynamic equilibrium $(p=n K T)$ with an additional constraint of either isothermality or adiabaticity; this gives the differential relationship

$$
d p=m c_{a}^{2} d n
$$

in lieu of a transport equation for energy. Note the limitation in this treatment of the plasma energy as it neglects any plasma heating due to laser absorption or damping of ponderomotively driven flows. For sufficiently high plasma temperatures, it is expected that these heating mechanisms are higher-order effects.

Under such assumptions, for an isothermal plasma, $c_{a}^{2}=K T / m$ is a constant isothermal sound speed, and for an adiabatic plasma, $p \propto n^{\gamma}$, and $c_{a}^{2}=\gamma K T / m$ is the isentropic sound speed. Taking $\gamma \rightarrow 1$ reproduces the isothermal result. Thus, without loss of generality, we will work with the adiabatic form and recover the isothermal result by setting $\gamma=1$.

The ponderomotive force $\mathbf{f}$ is a "light pressure" that pushes electrons (and subsequently ions via the electrostatic field) down gradients in light intensity. Since the ion-acoustic response of the plasma is a relatively slow phenomenon, 
we consider time scales much larger than the period of the time-harmonic light oscillation $2 \pi / \omega$. The ponderomotive force expressed as a time-averaged quantity over this period is

$$
\mathbf{f}_{p}=-\frac{Z e^{2}}{2 m_{e} m \omega^{2}} \nabla\left\langle|\mathcal{E}|^{2}\right\rangle_{\omega}=-F_{p} \nabla\left\langle|\mathcal{E}|^{2}\right\rangle_{\omega}
$$

where $m_{e}$ is the electron mass.

Direct consideration of the wave equation for the composite electric field $\mathcal{E}$ is unrealistic numerically, given the rapid temporal and spatial variation of the field relative to the scales of the flow evolution and laser beam widths. Therefore, defining $x$ to be the dominant direction of light propagation, $\mathcal{E}$ is assumed to be time-harmonic and enveloped about a transversely-averaged wavenumber $k \eta_{0}$,

$$
\mathcal{E}\left(t, x, \mathbf{x}_{\perp}\right)=\frac{1}{2} \mathcal{P}\left[E\left(t, x, \mathbf{x}_{\perp}\right) \exp \left\{-i \omega t+i k \bar{\eta}_{0}\left(x-x_{0}\right)\right\}+\text { c.c. }\right]
$$

where $\mathcal{P}$ is the constant polarization; $k=\omega / c$ is the free-space wavenumber; $c$ is the speed of light; $\eta_{0}(x)$ is the index of refraction averaged over the transverse directions $\mathbf{x}_{\perp}$; and

$$
\bar{\eta}_{0}=\frac{1}{x-x_{0}} \int_{x_{0}}^{x} \eta_{0}\left(x^{\prime}\right) d x^{\prime}
$$

is the index of refraction averaged over the distance $\left(x-x_{0}\right)$.

The index of refraction $\eta$ is related to the plasma density by $\eta^{2} \equiv 1-n / n_{c}$, where $n_{c}=\omega^{2} \epsilon_{0} m_{e} / e^{2}$ is the critical density. Further assuming one-way propagation at small angles to the longitudinal direction $x$, the complex-valued envelope $E$ will satisfy a paraxial wave equation in the $x$-direction,

$$
\partial_{x} E=\frac{i}{2 k \eta_{0}} \nabla_{\perp}^{2} E+\frac{i k^{2}}{2 k \eta_{0}}\left(\eta^{2}-\eta_{0}^{2}\right) E-\nu_{c} E
$$


where $\nu_{c}$ is a collisional absorption rate and $\nabla_{\perp}^{2}$ is the two-dimensional Laplacian over the transverse directions $\mathbf{x}_{\perp}$.

\subsection{Three-Wave Resonance}

We reduce the problem from the governing equations for the plasma (3) and electric field (8) to a set of coupled wave equations. The plasma flow field is assumed to be a perturbation imposed upon a large-scale background flow:

$$
n=n_{0}+\delta n, \quad \mathbf{v}=c_{s}\left(\mathbf{M}_{0}+\delta \mathbf{M}\right), \quad \text { and } \quad p=p_{0}+\delta p
$$

where $c_{s}$ is a characteristic sound speed. It is assumed that $|\delta n| \ll n_{0},|\delta p| \ll$ $p_{0}$, and $|\delta \mathbf{M}| \ll 1$. For either isentropic or adiabatic flow, it can be shown that substituting (9) into (4) and grouping terms of similar order leads to the relationships

$$
d p_{0} \approx m c_{0}^{2} d n_{0} \quad \text { and } \quad \delta p \approx m c_{0}^{2} \delta n
$$

where $c_{0}^{2}=\gamma p_{0} /\left(m n_{0}\right)$. Details are provided in Appendix A.

Before substituting (9) into (3) to derive perturbation equations, a form must be assumed for the damping force. This force is meant to represent the effect of linear Landau damping, a nonlocal phenomenon that is most easily expressed in Fourier space. Since the forcing of the plasma by the beat wave of the crossing laser beams will be confined to a narrow spectrum of wave numbers about $\left(\mathbf{k}_{0}-\mathbf{k}_{1}\right)$, a simpler model tuned to the correct Landau damping rate for that wavenumber band will be adopted. We choose

$$
\mathbf{d}(\mathbf{v})=-\nu_{a}\left(\mathbf{v}-\mathbf{v}_{0}\right)=-2 \nu_{a} c_{s} \delta \mathbf{M}
$$

that is, the damping (drag) force resists perturbations from the background 
flow. These perturbations relax to the background flow over a characteristic time $1 / \nu_{a}$, the inverse of the damping rate. ${ }^{1}$

Substituting (9), (10), and (11) into (3) and collecting terms of like order (neglecting products of small quantities) produces two sets of equations,

$$
\begin{array}{r}
\left(\partial_{t}+c_{s} \mathbf{M}_{0} \cdot \nabla\right) n_{0}+c_{s} n_{0} \nabla \cdot \mathbf{M}_{0}=0, \\
c_{s}\left(\partial_{t}+c_{s} \mathbf{M}_{0} \cdot \nabla\right) \mathbf{M}_{0}+c_{0}^{2} \nabla\left(\ln n_{0}\right)=0,
\end{array}
$$

and

$$
\begin{aligned}
&\left(\frac{1}{c_{s}} \partial_{t}+\mathbf{M}_{0} \cdot \nabla\right) \frac{\delta n}{n_{0}}+\nabla \cdot \delta \mathbf{M}=-\delta \mathbf{M} \cdot \nabla\left(\ln n_{0}\right)-2\left(\nabla \cdot \mathbf{M}_{0}\right) \frac{\delta n}{n_{0}}, \\
&\left(\frac{1}{c_{s}} \partial_{t}+\mathbf{M}_{0} \cdot \nabla+\frac{2 \nu_{a}}{c_{s}}\right) \delta \mathbf{M}+\frac{c_{0}^{2}}{c_{s}^{2}} \nabla \frac{\delta n}{n_{0}}= \\
& \frac{\mathbf{f}_{p}}{c_{s}^{2}}-(\gamma-1) \frac{c_{0}^{2}}{c_{s}^{2}} \nabla\left(\ln n_{0}\right) \frac{\delta n}{n_{0}}-\delta \mathbf{M} \cdot \nabla \mathbf{M}_{0}
\end{aligned}
$$

The first set of equations describes the evolution of the background flow, and the second describes the evolution of the perturbations driven by the ponderomotive force. In grouping terms, we have assumed that the ponderomotive force is suitably small that it contributes no leading-order effect on the background flow.

We now assume that perturbations possess a spatially harmonic factor. Defining the wavenumber of the acoustic perturbation to be $\mathbf{k}_{a}$, the perturbations

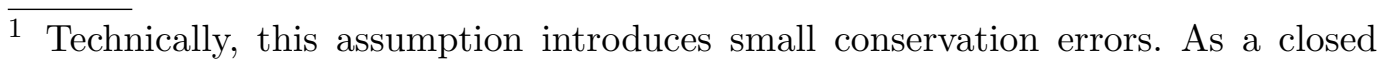
system, any momentum and energy lost by the ion acoustic wave through internal damping processes remains in the plasma and should appear in the background flow. However, these momentum and energy losses are small relative to the background flow energy and momentum and thus are reasonably negligible in our approximation. 
are

$$
\begin{aligned}
\frac{\delta n}{n_{0}} & =\frac{1}{2}(\widehat{n}+\text { c.c. })=\frac{1}{2}\left(\widetilde{n}(\mathbf{x}, t) \exp \left\{i \mathbf{k}_{a} \cdot \mathbf{x}\right\}+\text { c.c. }\right), \\
\delta \mathbf{M} & =\frac{1}{2}(\widehat{\mathbf{M}}+\text { c.c. })=\frac{1}{2}\left(\widetilde{\mathbf{M}}(\mathbf{x}, t) \exp \left\{i \mathbf{k}_{a} \cdot \mathbf{x}\right\}+\text { c.c. }\right) .
\end{aligned}
$$

Similarly, the electric field envelope satisfying (8) is assumed to be composed of two fields with harmonic factors:

$$
\begin{aligned}
E & =\widehat{E}_{0}(t, \mathbf{x})+\widehat{E}_{1}(t, \mathbf{x}) \\
& =\widetilde{E}_{0}(t, \mathbf{x}) \exp \left\{i \mathbf{k}_{0} \cdot \mathbf{x}\right\}+\widetilde{E}_{1}(t, \mathbf{x}) \exp \left\{i \mathbf{k}_{1} \cdot \mathbf{x}\right\}
\end{aligned}
$$

We define the primary harmonic factor of the light wave interaction to be

$$
\phi_{\Delta}(t, \mathbf{x}) \equiv \exp \left\{i\left(\mathbf{k}_{0}-\mathbf{k}_{1}\right) \cdot \mathbf{x}\right\}
$$

Substituting (15) into (5) and neglecting higher harmonics,

$$
\begin{aligned}
\mathbf{f}_{p} \approx-F \nabla\left[\frac{\left|\widehat{E}_{0}\right|^{2}+\left|\widehat{E}_{1}\right|^{2}}{2}+\left(\frac{\widetilde{E}_{0} \widetilde{E}_{1}^{*}}{2} \phi_{\Delta}(t, \mathbf{x})+\text { c.c. }\right)\right], \\
=-\frac{F}{2}\left[\nabla\left(\left|\widehat{E}_{0}\right|^{2}+\left|\widehat{E}_{1}\right|^{2}\right)\right. \\
\left.+\left(\left[\nabla\left(\widetilde{E}_{0} \widetilde{E}_{1}^{*}\right)+i\left(\mathbf{k}_{0}-\mathbf{k}_{1}\right) \widetilde{E}_{0} \widetilde{E}_{1}^{*}\right] \phi_{\Delta}(t, \mathbf{x})+\text { c.c. }\right)\right],
\end{aligned}
$$

Substituting (14) and (17b) into (13), and factoring out the harmonic variation 
of the acoustic perturbations,

$$
\begin{aligned}
\left(\frac{1}{c_{s}} \partial_{t}+i \mathbf{k}_{a} \cdot \mathbf{M}_{0}\right) \widetilde{n}+i \mathbf{k}_{a} \cdot \widetilde{\mathbf{M}}=-(2 \nabla & \left.\cdot \mathbf{M}_{0}+\mathbf{M}_{0} \cdot \nabla\right) \widetilde{n}-\frac{1}{n_{0}} \nabla \cdot\left[n_{0} \widetilde{\mathbf{M}}\right] \\
\left(\frac{1}{c_{s}} \partial_{t}+i \mathbf{k}_{a} \cdot \mathbf{M}_{0}+\frac{2 \nu_{a}}{c_{s}}\right) \widetilde{\mathbf{M}}+i \frac{c_{0}^{2}}{c_{s}^{2}} \mathbf{k}_{a} \widetilde{n} & \left.-\left((\gamma-1) \frac{c_{0}^{2}}{c_{s}^{2}} \nabla\left(\ln n_{0}\right)+\frac{c_{0}^{2}}{c_{s}^{2}} \nabla\right) \widetilde{n}\right) \\
& -\mathbf{M}_{0} \cdot \nabla \widetilde{\mathbf{M}}-\widetilde{\mathbf{M}} \cdot \nabla \mathbf{M}_{0} \\
& -\frac{F}{c_{s}^{2}} \exp \left\{-i \mathbf{k}_{a} \cdot \mathbf{x}\right\} \nabla\left(\left|\widehat{E}_{0}\right|^{2}+\left|\widehat{E}_{1}\right|^{2}\right) \\
& -\frac{F}{c_{s}^{2}}\left[\nabla\left(\widetilde{E}_{0} \widetilde{E}_{1}^{*}\right)+i\left(\mathbf{k}_{0}-\mathbf{k}_{1}\right) \widetilde{E}_{0} \widetilde{E}_{1}^{*}\right],
\end{aligned}
$$

where we have assumed the matching condition $\mathbf{k}_{0}=\mathbf{k}_{1}+\mathbf{k}_{a}$. To identify the dominant terms, we must consider the various length scales in a characteristic problem.

Several natural length scales can be distinguished. The background flow imposes a velocity length scale defined by

$$
\mathbf{L}_{\perp}(t, \mathbf{x}) \equiv\left(\nabla \cdot \mathbf{M}_{0}\right)^{-1} \mathbf{M}_{0}
$$

as well as a domain width $L$. The laser beams impose several length scales, most notably a width or diameter $D_{0,1}(t, \mathbf{x})$ and the dominant wavelength, $2 \pi / k$. In addition, the interaction between the two beams propagating at an angle produces, through the ponderomotive force, a density wave at length $2 \pi /\left|k_{0}-k_{1}\right|=2 \pi /\left|k_{a}\right|$, which is longer than the light wavelength. Another intermediate length is the speckle scale length, where speckles in the beam are caused by the lack of coherence within each beam. The speckle scale has a characteristic length of $L_{s}=f 2 \pi / k_{0}$ where the $f$-number, $f=f_{l} / D$, is the ratio of the focal length to aperture of the lens. To distinguish between the density wave scale and the speckle scale, we can restrict the angle between the 
beams, $2 \theta$, to be greater the the beam divergence angle, $\theta_{f}=\tan ^{-1}(1 / 2 f)$. Physically, we are making a distinction between energy exchanges between beams and similar internal interactions that modify the properties of each beam that would occur even if the other beam were not present.

We limit our consideration to the interaction of two idealized beams with smooth spatial envelopes, associating the scale of the beam envelope with $D_{0,1}$ and neglecting the speckle scale length. We further specialize to the case where the velocity scale length, plasma domain length, and beam diameters are all of roughly the same order of magnitude, $\left|\mathbf{L}_{\perp}\right| \sim L \sim D$, and the laser wavelengths are much smaller than these lengths, e.g., $k\left|\mathbf{L}_{\perp}\right| \gg 1$. Finally, we identify the perturbation scale length $2 \pi /\left|\mathbf{k}_{a}\right|$ as an intermediate scale such that $\left|\mathbf{k}_{a} \cdot \mathbf{L}_{\perp}\right| \gg 1 \gg\left|\mathbf{k}_{a}\right| / k$.

Having identified the relative sizes of the various scale lengths, we reconsider (18). Clearly, terms that vary with derivatives of the background flow or the envelopes will be much smaller than terms with factors proportional to $\left|\mathbf{k}_{a}\right|$. Neglecting such terms we find

$$
\begin{gathered}
\left(\frac{1}{c_{s}} \partial_{t}+i \mathbf{k}_{a} \cdot \mathbf{M}_{0}\right) \widetilde{n}+i \mathbf{k}_{a} \cdot \widetilde{\mathbf{M}} \approx 0 \\
\left(\frac{1}{c_{s}} \partial_{t}+i \mathbf{k}_{a} \cdot \mathbf{M}_{0}+\frac{2 \nu_{a}}{c_{s}}\right) \widetilde{\mathbf{M}}+i \frac{c_{0}^{2}}{c_{s}^{2}} \mathbf{k}_{a} \widetilde{n} \approx-\frac{i F}{c_{s}^{2}} \mathbf{k}_{a} \widetilde{E}_{0} \widetilde{E}_{1}^{*},
\end{gathered}
$$

which is a nonlinearly forced system of ODEs with variable coefficients. Multiplying through by $\exp \left\{i \mathbf{k}_{a} \cdot \mathbf{x}\right\}$ and dividing by $\left|\mathbf{k}_{a}\right|$, this system (20) can also be written as

$$
\begin{gathered}
\left(\frac{1}{i \omega_{a}} \partial_{t}+\frac{\mathbf{k}_{a}}{\left|\mathbf{k}_{a}\right|} \cdot \mathbf{M}_{0}\right) \widehat{n}+\frac{\mathbf{k}_{a}}{\left|\mathbf{k}_{a}\right|} \cdot \widehat{\mathbf{M}}=0 \\
\left(\frac{1}{i \omega_{a}} \partial_{t}+\frac{\mathbf{k}_{a}}{\left|\mathbf{k}_{a}\right|} \cdot \mathbf{M}_{0}-\frac{i 2 \nu_{a}}{\omega_{a}}\right) \widehat{\mathbf{M}}+\frac{c_{0}^{2}}{c_{s}^{2}} \frac{\mathbf{k}_{a}}{\left|\mathbf{k}_{a}\right|} \widehat{n} \approx-\frac{Z e^{2} \widehat{E}_{0} \widehat{E}_{1}^{*}}{2 m_{e} m \omega^{2} c_{s}^{2}} \frac{\mathbf{k}_{a}}{\left|\mathbf{k}_{a}\right|}
\end{gathered}
$$


All that remains is to specify the nonlinear forcing due to the beating of the light waves.

To derive the reduced equations for the light, we begin with the paraxial equation (8). Since the density has the form $n=n_{0}+\delta n$, the difference in indices of refraction is

$$
\eta^{2}-\eta_{0}^{2}=\frac{\bar{n}_{0}-n_{0}-\delta n}{2 \eta_{0} n_{c}}
$$

so (8) becomes

$$
\left[\partial_{x}-\frac{i}{2 k \eta_{0}} \nabla_{\perp}^{2}-\frac{i k}{2 \eta_{0}} \frac{\left(\bar{n}_{0}-n_{0}\right)}{n_{c}}+\nu_{c}+\frac{i k}{2 \eta_{0}} \frac{\delta n}{n_{c}}\right] E=0
$$

We write $\delta n=n_{0}\left(\widehat{n}+\widehat{n}^{*}\right) / 2$ and use the assumed form (15a), to obtain

$$
\left[\partial_{x}-\frac{i}{2 k \eta_{0}} \nabla_{\perp}^{2}-\frac{i k}{2 \eta_{0}} \frac{\left(\bar{n}_{0}-n_{0}\right)}{n_{c}}+\nu_{c}+\frac{i k^{2} n_{0}}{4 k \eta_{0}} \frac{\left(\widehat{n}+\widehat{n}^{*}\right)}{n_{c}}\right]\left(\widehat{E}_{0}+\widehat{E}_{1}\right)=0 .
$$

Introducing the harmonic factors from (14) and (15b) and neglecting the higher harmonics resulting from multiplication, we group terms by like harmonic variation in $\mathbf{k}_{0}$ or $\mathbf{k}_{1}$. Each of these collections must equal zero independently, and we arrive at a pair of coupled mode equations:

$$
\begin{aligned}
& {\left[\partial_{x}-\frac{i}{2 k \eta_{0}} \nabla_{\perp}^{2}-\frac{i k}{2 \eta_{0}} \frac{\left(\bar{n}_{0}-n_{0}\right)}{n_{c}}+\nu_{c}\right] \widehat{E}_{0}=-\frac{i k}{4 \eta_{0}} \frac{n_{0}}{n_{c}} \widehat{n} \widehat{E}_{1},} \\
& {\left[\partial_{x}-\frac{i}{2 k \eta_{0}} \nabla_{\perp}^{2}-\frac{i k}{2 \eta_{0}} \frac{\left(\bar{n}_{0}-n_{0}\right)}{n_{c}}+\nu_{c}\right] \widehat{E}_{1}=-\frac{i k}{4 \eta_{0}} \frac{n_{0}}{n_{c}} \widehat{n}^{*} \widehat{E}_{0} .}
\end{aligned}
$$

The source terms clearly show the coupling between the light fields through the density perturbation.

The strong-damping or steady-state resonance in the system (21) and (25) is found by assuming that the temporal derivatives of (21) are small compared 
to the damping terms. Formally,

$$
\begin{gathered}
\frac{\mathbf{k}_{a}}{\left|\mathbf{k}_{a}\right|} \cdot \mathbf{M}_{0} \widehat{n}+\frac{\mathbf{k}_{a}}{\left|\mathbf{k}_{a}\right|} \cdot \widehat{\mathbf{M}} \approx 0 \\
\left(\frac{\mathbf{k}_{a}}{\left|\mathbf{k}_{a}\right|} \cdot \mathbf{M}_{0}-\frac{i 2 \nu_{a}}{\omega_{a}}\right) \widehat{\mathbf{M}}+\frac{c_{0}^{2}}{c_{s}^{2}} \frac{\mathbf{k}_{a}}{\left|\mathbf{k}_{a}\right|} \widehat{n} \approx-\frac{Z e^{2} \widehat{E}_{0} \widehat{E}_{1}^{*}}{2 m_{e} m \omega^{2} c_{s}^{2}} \frac{\mathbf{k}_{a}}{\left|\mathbf{k}_{a}\right|} .
\end{gathered}
$$

Considering the component of the momentum equation parallel to the acoustic wavenumber and solving for the density perturbation,

$$
\widehat{n}=\frac{1}{2} \frac{Z K T_{e}}{m c_{s}^{2}} \frac{\widehat{v}_{0} \widehat{v}_{1}^{*}}{v_{e}^{2}}\left[\frac{\left|\mathbf{k}_{a} \cdot \mathbf{M}_{0}\right|^{2}}{\left|\mathbf{k}_{a}\right|^{2}}-\frac{c_{0}^{2}}{c_{s}^{2}}-i \frac{2 \nu_{a}}{\omega_{a}} \frac{\mathbf{k}_{a}}{\left|\mathbf{k}_{a}\right|} \cdot \mathbf{M}_{0}\right]^{-1}
$$

where $\widehat{v}_{j} \equiv e \widehat{E}_{j} / m_{e} \omega(j=0,1)$ are the (complex) jitter velocities due to the pump and probe beams, respectively, and the electron thermal speed is $v_{e}=$ $\sqrt{K T_{e} / m_{e}}$. The resonance is clear by inspection: when the flow velocity parallel to the acoustic wave vector is sonic, that is $c_{s}^{2}\left|\mathbf{k}_{a} \cdot \mathbf{M}_{0}(t, \mathbf{x})\right|^{2}=c_{0}^{2}(t, \mathbf{x})\left|\mathbf{k}_{a}\right|^{2}$, the real part of the denominator vanishes, leaving only the damping term to limit the amplitude of the acoustic perturbation.

How rapidly this steady state develops depends on the time scale of the background flow. In general, the background flow evolves slowly enough that its effects on the beam propagation and hence the acoustic wave excitation are small. The steady state then will be reached in a time on the order of the inverse damping rate $1 / \nu_{a}$, as this is the characteristic time over which the transients in the initial growth of the acoustic waves will damp away. However, when the background flow causes sufficiently rapid motion of the beams (e.g., , $\left.\partial_{t} \widehat{n} / \nu_{a}=O(1)\right)$, the resonance (27) is modified. This point was demonstrated in previous work $[19,20]$ that modified the Rosenbluth gain formula [18] to account for effects of time-dependent background flows.

We consider the case where the motion of the beams due to the background flow evolution causes a temporal phase mismatch. For fixed beams, the pon- 
deromotive forcing is stationary in the the laboratory frame. From the frame of a particle moving with the fluid, the forcing appears to have frequency of $\mathbf{k}_{a} \cdot \mathbf{M}_{0} c_{s}$. However, if the beams are moving with velocity $\mathbf{v}$ in the direction of the flow due to refraction, the fluid particle would instead see a forcing frequency of $\mathbf{k}_{a} \cdot\left(\mathbf{M}_{0} c_{s}-\mathbf{v}\right)$, which shifts the resonance.

We explicitly identify this rapid variation in the acoustic response, that is,

$$
\widehat{n}(t, \mathbf{x})=\check{n}(t, \mathbf{x}) \exp \left\{i \phi_{a}(t, \mathbf{x})\right\}
$$

and assume that this matches the effective phase variation in the ponderomotive forcing. This is in spirit similar to the approach taken in $[19,20]$, although we neglect any wavenumber mismatch $\nabla \phi_{a}$ in favor of frequency mismatch $\partial_{t} \phi_{a}$. We anticipate that for large flow gradients $\partial_{t} \phi_{a} \sim \nu_{a}$ and therefore is not negligible, while $\left|\nabla \phi_{a}\right| \ll\left|\mathbf{k}_{a}\right|$ and thus can be neglected to leading order.

Substituting this into (21) and assuming that time variation of $\check{n}$ is slow, one finds the modified resonance condition

$$
\begin{aligned}
\check{n}=\frac{Z K T_{e}}{2 m c_{s}^{2}} \frac{\widehat{v}_{0} \widehat{v}_{1}^{*}}{v_{e}^{2}}\left[\frac{\left|\mathbf{k}_{a} \cdot \mathbf{M}_{0}\right|^{2}}{\left|\mathbf{k}_{a}\right|^{2}}-\frac{c_{0}^{2}}{c_{s}^{2}}\right. & +\frac{\dot{\phi}_{a}}{\omega_{a}} \frac{2 \mathbf{k}_{a} \cdot \mathbf{M}_{0}}{\left|\mathbf{k}_{a}\right|} \\
& \left.-i\left\{\frac{2 \nu_{a}}{\omega_{a}}\left(\frac{\mathbf{k}_{a} \cdot \mathbf{M}_{0}}{\left|\mathbf{k}_{a}\right|}+\frac{\dot{\phi}_{a}}{\omega_{a}}\right)+\frac{\ddot{\phi}_{a}}{\omega_{a}^{2}}\right\}\right]^{-1}
\end{aligned}
$$

where $\dot{\phi}_{a}$ and $\ddot{\phi}_{a}$ denote first and second time derivatives of the frequency mismatch and where nonlinear terms in $\dot{\phi}_{a}$ have been neglected. For nonzero frequency mismatch, the effect is to shift the location of resonance away from the sonic condition and to alter the effective damping, which adjusts the maximum amplitude of the acoustic response. For the numerical simulations presented in Section 4, the frequency mismatch will only be an issue for flows with short scale lengths. 


\subsection{Two-Dimensional Simplifications}

We now restrict consideration to two dimensions with a one-dimensional background flow transverse to the primary direction of beam propagation as shown in Figure 1. We assume a configuration in which the excited ion acoustic wave is nearly aligned with the transverse direction $y$. For beams initially at small, equal and opposite angles to the direction of propagation, this is a good approximation, even with the inclusion of beam bending due to transverse density gradients. Thus, we have

$$
\mathbf{M}_{0}+\frac{1}{2}\left[\widehat{\mathbf{M}}+\widehat{\mathbf{M}}^{*}\right]=\left(M_{0}(t, y)+\frac{1}{2}\left[\widehat{M}(t, \mathbf{x})+\widehat{M}^{*}(t, \mathbf{x})\right]\right) \hat{\mathbf{e}}_{y}
$$

and $\mathbf{k}_{a} \approx k_{a y} \hat{\mathbf{e}}_{y}$ since $\left|k_{a y}\right| \gg\left|k_{a x}\right|$. Similarly, $\left|k_{0 y}\right| \gg\left|k_{0 x}\right|,\left|k_{1 y}\right| \gg\left|k_{1 x}\right|$, and $\left|k_{0 y}\right| \approx-\left|k_{1 y}\right|$. We therefore take $\mathbf{k}_{0} \approx k_{0 y} \hat{\mathbf{e}}_{y} \equiv-k_{\perp} \hat{\mathbf{e}}_{y}$ and $\mathbf{k}_{1} \approx k_{1 y} \hat{\mathbf{e}}_{y} \equiv k_{\perp} \hat{\mathbf{e}}_{y}$ such that $k_{a y}=-2 k_{\perp}$. Our perturbation (21) and coupled mode equations (25) then reduce to

$$
\begin{gathered}
\left(\frac{1}{i \omega_{a}} \partial_{t}-M_{0}\right) \widehat{n}-\widehat{M} \approx 0, \\
\left(\frac{1}{i \omega_{a}} \partial_{t}-M_{0}-\frac{i 2 \nu_{a}}{\omega_{a}}\right) \widehat{M}-\frac{c_{0}^{2}}{c_{s}^{2}} \widehat{n} \approx \frac{Z e^{2} \widehat{E}_{0} \widehat{E}_{1}^{*}}{2 m m_{e} m \omega^{2} c_{s}^{2}}, \\
{\left[\partial_{x}-\frac{i}{2 k \eta_{0}} \nabla_{\perp}^{2}-\frac{i k}{2 \eta_{0}} \frac{\left(\bar{n}_{0}-n_{0}\right)}{n_{c}}+\nu_{c}\right] \widehat{E}_{0} \approx-\frac{i k}{4 \eta_{0}} \frac{n_{0}}{n_{c}} \widehat{n} \widehat{E}_{1},} \\
{\left[\partial_{x}-\frac{i}{2 k \eta_{0}} \nabla_{\perp}^{2}-\frac{i k}{2 \eta_{0}} \frac{\left(\bar{n}_{0}-n_{0}\right)}{n_{c}}+\nu_{c}\right] \widehat{E}_{1} \approx-\frac{i k}{4 \eta_{0}} \frac{n_{0}}{n_{c}} \widehat{n}^{*} \widehat{E}_{0} .}
\end{gathered}
$$

To close this system, a one-dimensional background flow must be specified.

For lasers crossing near the entry hole of a hohlraum, the plasma flow from the interior of the hohlraum will be expanding out into a near vacuum. A simple analytical model of an expanding flow that satisfies the leading-order system (12) is the one-dimensional, self-similar rarefaction. The leading-order 
system (12) together with the conditions

$$
n_{0}(y=0, t)=\alpha n_{c}, \quad \lim _{y \rightarrow \infty} n_{0}(y, t)=0, \quad \text { and, } \forall y, t, \quad n_{0}(y, t)>0,
$$

possesses a unique similarity solution, which for $y / L_{\perp}<2 /(\gamma-1)$, is

$$
\begin{array}{cc}
n_{0}(y, t)=\alpha n_{c}\left(1-\frac{\gamma-1}{2} \frac{y}{L_{\perp}}\right)^{2 /(\gamma-1)}, & M_{0}(y, t)=1+\frac{y}{L_{\perp}}, \\
c_{0}^{2}(y, t)=\frac{\gamma p_{0}}{n_{0}}=c_{s}^{2}\left(1-\frac{\gamma-1}{2} \frac{y}{L_{\perp}}\right)^{2}, & L_{\perp}(t)=L_{\perp}(0)+\frac{\gamma+1}{2} c_{s} t,
\end{array}
$$

where the reference sound speed $c_{s}$ is now defined to be the sound speed at the sonic line, positioned at $y=0$. The isothermal result is obtained by taking the limit as $\gamma \rightarrow 1$ and significantly simplifies to $c_{0} \equiv c_{s}$ and $n_{0}=$ $\alpha n_{c} \exp \left(-y / L_{\perp}\right)$. Note that the adiabatic $(\gamma>1)$ number density vanishes at a finite distance from the center line, beyond which it is assumed to be zero.

\subsection{Steady-State Gain Prediction}

[Fig. 2 about here.]

If we simplify the geometry and physics of the problem even further, we can derive an upper-bound, analytic, steady-state gain estimate of the resonant interaction. We neglect pump depletion, diffraction, and refraction due to the $n_{0}(y, t)$ density gradient, and we assume that the beams are positioned symmetrically at angles $\pm \theta$ about the sonic line, as demonstrated in Figure 2. The resulting equations for the enveloped light amplitudes are

$$
\begin{aligned}
& {\left[\cos \theta \partial_{x}-\sin \theta \partial_{y}+\nu_{c} \cos \theta\right] \widetilde{E}_{0} \approx 0} \\
& {\left[\cos \theta \partial_{x}+\sin \theta \partial_{y}+\nu_{c} \cos \theta\right] \widetilde{E}_{1} \approx-\frac{i k \cos \theta}{4 \eta_{0}} \frac{n_{0}}{n_{c}} \widetilde{n}^{*} \widetilde{E}_{0}}
\end{aligned}
$$

where we have identified $k_{0 x}=k_{1 x l}=k \eta_{0} \cos \theta$ and $-k_{0 y}=k_{1 y}=k \eta_{0} \sin \theta$. 
Note that the wave equation for the pump beam has decoupled from the system, but there still exists a nonlinear coupling between the probe beam and acoustic response.

Rotating our coordinate system so that it lies along the probe beam (see Appendix B), the PDE (34b) reduces to the ODE

$$
\left.\left[\frac{d}{d \zeta}+\nu_{c} \cos \theta\right] \widetilde{E}_{1}=-\frac{i k \cos \theta}{4 \eta_{0}} \frac{n_{0}}{n_{c}} \widetilde{n}^{*} \widetilde{E}_{0}, \quad \text { (on lines of constant } \xi\right)
$$

where $\zeta=y \cos \theta+x \sin \theta$ and $\xi=y \cos \theta-x \sin \theta$. Substituting in the steadystate density perturbation (27),

$$
\begin{aligned}
{\left[\frac{d}{d \zeta}+\nu_{c} \cos \theta\right] \widetilde{E}_{1} } & =\frac{k \cos \theta}{8 \eta_{0}} \frac{n_{0}}{n_{c}} \frac{Z K T_{e}}{m c_{s}^{2}} \frac{\left|\widehat{v}_{0}\right|^{2}}{v_{e}^{2}}\left[\frac{2 \nu_{a}}{\omega_{a}} M_{0}+i\left(M_{0}^{2}-\frac{c_{0}^{2}}{c_{s}^{2}}\right)\right]^{-1} \widehat{E}_{1} \\
& \equiv \frac{k \cos \theta}{8 \eta_{0}} \frac{n_{0}}{n_{c}} \frac{Z K T_{e}}{m c_{s}^{2}} \frac{\left|\widehat{v}_{0}\right|^{2}}{v_{e}^{2}} \Gamma(\zeta, \xi) \widetilde{E}_{1}
\end{aligned}
$$

Integrating across the interaction region (from $\zeta_{0}$ to $\zeta_{1}$ ),

$$
\begin{aligned}
\widetilde{E}_{1}\left(\zeta_{1}, \xi\right)=\widetilde{E}_{1}\left(\zeta_{0}, \xi\right) \exp \left[-\nu_{c}\left(\zeta_{1}-\zeta_{0}\right)\right. & \\
& \left.+\int_{\zeta_{0}(\xi)}^{\zeta_{1}(\xi)} \frac{k \cos \theta}{8 \eta_{0}} \frac{n_{0}}{n_{c}} \frac{Z K T_{e}}{m c_{s}^{2}} \frac{\left|\widehat{v}_{0}\right|^{2}}{v_{e}^{2}} \Gamma\left(\zeta^{\prime}, \xi\right) d \zeta^{\prime}\right]
\end{aligned}
$$

where $\zeta_{0}(\xi)$ is the point at which interaction begins for a given $\xi$ and $\zeta_{1}(\xi)$ the point at which interaction ends.

[Fig. 3 about here.]

As shown in Figure 3, these upper and lower limits of integration are easily found in this simplified geometry; the derivation is presented in Appendix C.

The gain along a ray of the probe through the interaction region is defined to 
be

$$
\begin{aligned}
g(\xi) & \equiv\left|\frac{\widetilde{E}_{1}\left(\zeta_{1}(\xi), \xi\right)}{\widetilde{E}_{1}\left(\zeta_{0}(\xi), \xi\right)}\right| \\
& =\exp \left[-\nu_{c}\left(\zeta_{1}-\zeta_{0}\right)+\operatorname{Re}\left\{\int_{\zeta_{0}(\xi)}^{\zeta_{1}(\xi)} \frac{k \cos \theta}{8 \eta_{0}} \frac{n_{0}}{n_{c}} \frac{Z K T_{e}}{m c_{s}^{2}} \frac{\left|\widehat{v}_{0}\right|^{2}}{v_{e}^{2}} \Gamma\left(\zeta^{\prime}, \xi\right) d \zeta^{\prime}\right\}\right]
\end{aligned}
$$

Assuming that the pump beam remains constant through the interaction region, the integral in (38) can be computed analytically for the self-similar background flow prescribed by (33). This derivation is presented in Appendix D. For the isothermal case, the result of the integration is simply

$$
\operatorname{Re}\left\{\int_{\zeta_{0}(\xi)}^{\zeta_{1}(\xi)} \Gamma\left(\zeta^{\prime}, \xi\right) d \zeta^{\prime}\right\}=\left.\frac{L_{\perp} \psi_{r}}{2 \sin \theta} \arctan \left[\psi_{r} \frac{\left(\nu_{a} / \omega_{a}\right)^{2}+X(1+X / 2)}{\nu_{a} / \omega_{a}}\right]\right|_{X_{0}(\xi)} ^{X_{1}(\xi)}
$$

where

$$
X_{0}=\frac{\xi-D / 2}{2 L_{\perp} \cos \theta} \quad \text { and } \quad X_{1}=\frac{\xi+D / 2}{2 L_{\perp} \cos \theta} .
$$

When $D \omega_{a} /\left(L_{\perp} \nu_{a}\right) \ll 1$, it can be shown that the argument of the arctan is small, and so

$$
\begin{aligned}
\operatorname{Re}\left\{\int_{\zeta_{0}(\xi)}^{\zeta_{1}(\xi)} \Gamma\left(\zeta^{\prime}, \xi\right) d \zeta^{\prime}\right\} & \approx \frac{L_{\perp}}{2 \sin \theta} \frac{\psi_{r}^{2}}{\nu_{a} / \omega_{a}}\left[X_{1}\left(1+X_{1} / 2\right)-X_{0}\left(1+X_{0} / 2\right)\right] \\
& =\frac{L_{\perp}}{2 \sin \theta} \frac{\psi_{r}^{2}}{\nu_{a} / \omega_{a}} \frac{D}{2 L_{\perp} \cos \theta}\left[1+\frac{\xi}{2 L_{\perp} \cos \theta}\right] \\
& =\frac{D}{2 \nu_{a} / \omega_{a} \sin 2 \theta} \frac{1}{1-\left(\nu_{a} / \omega_{a}\right)^{2}}\left[1+\frac{\xi}{2 L_{\perp} \cos \theta}\right] \\
& \approx \frac{D}{2 \nu_{a} / \omega_{a} \sin 2 \theta} \frac{1}{1-\left(\nu_{a} / \omega_{a}\right)^{2}}
\end{aligned}
$$

since $0 \leq|2 \xi| \leq D$. Thus the gain is

$$
g(\xi)=\exp \left[-\nu_{c}\left(\zeta_{1}-\zeta_{0}\right)+\frac{k}{32 \eta_{0}} \frac{n_{0}}{n_{c}} \frac{Z K T_{e}}{m c_{s}^{2}} \frac{\left|\widehat{v}_{0}\right|^{2}}{v_{e}^{2}} \frac{D \omega_{a}}{\nu_{a} \sin \theta} \frac{\omega_{a}^{2}}{\omega_{a}^{2}-\nu_{a}^{2}}\right]
$$

which is independent of the velocity scale length $L_{\perp}$. 


\section{$3 \quad$ Numerical Implementation}

In this section, we describe two numerical implementations of the theoretical models presented in Section 2. The first is a numerical evaluation of the strongly-damped, steady-state gain formula (38), which makes use of a ray-tracing algorithm to estimate the interaction region of the two laser beams. The more sophisticated, time-dependent model is a discretization of the differential-algebraic system of coupled mode equations (31).

\subsection{Steady-State Analytic Model (SSA)}

A numerical model for steady-state energy transfer can be obtained using the formula (38) for the probe gain along rays. Approximating the interaction region $\zeta_{0}(\xi) \leq \zeta \leq \zeta_{1}(\xi),-D / 2 \leq \xi \leq D / 2$ by a parallelogram, the probe gain can be computed using a numerical quadrature to evaluate the integral (38) along equispaced discrete rays $\xi_{n}$, then summing the results.

For a plasma with uniform density, the calculation of the interaction region is a straightforward trigonometry exercise detailed in Appendix C. In the case of a non-uniform density, such as in the rarefaction fan considered here, the corresponding variation of the refractive index causes a deflection of the beams that must be taken into account in the calculation of the interaction region. This can be accomplished by ray-tracing the beam "edges" (given, e.g., , by the FWHM diameter for a Gaussian profile) to determine the boundaries of the deflected interaction region. This procedure, which is described in Appendix E, involves the numerical integration of a small system of ordinary differential equations. 
The applicability of this semi-analytic, steady-state gain model is limited by the various assumptions required for the derivation of the gain formula (38). By numerically approximating the gain integral, a few of these assumptions can be partially relaxed. For example, instead of assuming perfectly flat beams, approximate pump and probe profiles can be incorporated in the numerical quadrature. A limited amount of pump depletion can also be included by wrapping an iteration loop around the gain calculation, allowing the pump energy to relax to a more self-consistent value.

We have implemented this steady-state model in a Matrab [21] script. The ray-tracing uses the provided explicit fourth-order Runge-Kutta method to compute the instantaneous beam interaction region for the specified background density. As we will show in the next section, predictions by this method can be fairly accurate in some regimes. In other cases, however, beam propagation effects and unsteady ion acoustic effects can result in substantial overestimates of gain. For such problems, a more physically realistic model is required.

\subsection{Time-Dependent Couple Mode Model (TDCM)}

The coupled mode system (31) is a more complete model of the light propagation and unsteady acoustic response. Considering a uniform grid with cells of size $h_{x} \times h_{y}$ indexed by $\left\{(j, m): j=1,2, \ldots, N, m=1,2, \ldots, N_{\perp}\right\}$, we construct a spatial semi-discretization of (31) for the values of the dependent variables at the $x$-face centers, i.e., the cell faces perpendicular to the $x$ direction. In particular, let $\widehat{n}_{j, m}, \widehat{M}_{j, m}, \widehat{E}_{0, j, m}$ and $\widehat{E}_{1, j, m}$ denote the values of $\widehat{n}, \widehat{M}$, $\widehat{E}_{0}$ and $\widehat{E}_{1}$ at the center of the right-hand $x$-face of cell $(j, m)$ (or left-hand domain boundary if $j=0$ ), respectively. 
Consequently, the ordinary differential equations for the plasma perturbations (31a) and (31b) can be collocated at the $x$-face centers. The resulting equations are

$$
\begin{gathered}
\left(\frac{1}{i \omega_{a}} \partial_{t}-M_{0, m}\right) \widehat{n}_{j, m}-\widehat{M}_{j, m}=0 \\
\left(\frac{1}{i \omega_{a}} \partial_{t}-M_{0, m}-\frac{i 2 \nu_{a}}{\omega_{a}}\right) \widehat{M}_{j, m}-\frac{c_{0, m}^{2}}{c_{s}^{2}} \widehat{n}_{j, m}-\frac{Z e^{2} \widehat{E}_{0, j, m} \widehat{E}_{1, j, m}^{*}}{2 m_{e} m \omega^{2} c_{s}^{2}}=0
\end{gathered}
$$

for $j=0, \ldots, N$ and $m=1, \ldots, N_{\perp}$. In the absence of ponderomotive excitation, there are no plasma perturbations, so the initial conditions for the perturbation amplitudes are $\widehat{n}_{j, m}(t=0)=\widehat{M}_{j, m}(t=0)=0$ for each $j$ and $m$. Let $\eta_{0} \equiv \sqrt{1-\sum_{m=1}^{N_{\perp}} n_{0, m}(t) / n_{c} N_{\perp}}$ denote the refractive index computed from the discrete transverse average of the background density $n_{0}$ defined in (33), and let $\nu_{c, m}, m=1, \ldots, N_{\perp}$, denote the discrete spatial absorption frequency. We discretize the pump equation (31c) as

$$
\begin{aligned}
\frac{\widehat{E}_{0, j, m}-\widehat{E}_{0, j-1, m}}{h_{x}}-\frac{i}{4 k \eta_{0} h_{y}^{2}}\left[\left(\widehat{E}_{0, j-1, m-1}+\widehat{E}_{0, j, m-1}\right)\right. & \left.-2\left(\widehat{E}_{0, j-1, m}+\widehat{E}_{0, j, m}\right)+\left(\widehat{E}_{0, j-1, m+1}+\widehat{E}_{0, j, m+1}\right)\right] \\
& +\frac{1}{2}\left[\frac{i k}{2 \eta_{0}} \frac{\left(n_{0, m}-\bar{n}_{0}\right)}{n_{c}}+\nu_{c, m}\right]\left(\widehat{E}_{0, j-1, m}+\widehat{E}_{0, j, m}\right) \\
& +\frac{i k}{16 \eta_{0}} \frac{\widehat{n}_{j-1, m}+\widehat{n}_{j, m}}{n_{c}}\left(\widehat{E}_{1, j-1, m}+\widehat{E}_{1, j, m}\right)=0
\end{aligned}
$$

and, analogously, the probe equation (31d) as

$$
\begin{aligned}
\frac{\widehat{E}_{1, j, m}-\widehat{E}_{1, j-1, m}}{h_{x}}-\frac{i}{4 k \eta_{0} h_{y}^{2}}\left[\left(\widehat{E}_{1, j-1, m-1}+\widehat{E}_{1, j, m-1}\right)\right. & \\
& \left.-2\left(\widehat{E}_{1, j-1, m}+\widehat{E}_{1, j, m}\right)+\left(\widehat{E}_{1, j-1, m+1}+\widehat{E}_{1, j, m+1}\right)\right] \\
& +\frac{1}{2}\left[\frac{i k}{2 \eta_{0}} \frac{\left(n_{0, m}-\bar{n}_{0}\right)}{n_{c}}+\nu_{c, m}\right]\left(\widehat{E}_{1, j-1, m}+\widehat{E}_{1, j, m}\right) \\
& +\frac{i k}{16 \eta_{0}} \frac{\widehat{n}_{j-1, m}^{*}+\widehat{n}_{j, m}^{*}}{n_{c}}\left(\widehat{E}_{0, j-1, m}+\widehat{E}_{0, j, m}\right)=0
\end{aligned}
$$


for $j=1, \ldots, N$ and $m=1, \ldots, N_{\perp}$. In (44) and (45), we have applied the standard central differencing of the transverse Laplacian $\nabla_{\perp}^{2}$. As explained in [17], the Crank-Nicholson-type discretization in the $x$ direction ensures that energy is algebraically conserved by the algorithm except for losses due to absorption.

The paraxial equations are one-way wave equations, that is, they are hyperbolic with $x$ as the time-like variable. Accordingly, the incident beam conditions at the left-hand boundary, $\widehat{E}_{0,0, m}$ and $\widehat{E}_{1,0, m}, m=1, \ldots, N_{\perp}$, are initial conditions and are prescribed by specifying the beam cross sections and propagation angles relative to the $x$-direction. Since the simulation domain is artificially truncated, to obtain a well-posed problem, boundary conditions must be imposed in the transverse direction by prescribing $\widehat{E}_{0, j, 0}, \widehat{E}_{0, j, N_{\perp}+1}, \widehat{E}_{1, j, 0}$, $\widehat{E}_{1, j, N_{\perp}+1}$ for $j=1, \ldots, N$. We assume that the fields have zero amplitude on these artificial transverse boundaries, which is adequate so long as the beams do not reach the transverse boundaries.

The semi-discretized equations (43a)-(45), together with boundary conditions, comprise a differential-algebraic system that can be formally expressed as

$$
\mathbf{f}\left(t, \mathbf{u}(t), \mathbf{u}^{\prime}(t)\right)=\mathbf{0}
$$


where

$$
\mathbf{u}(t) \equiv\left(\begin{array}{c}
\left\{\widehat{n}_{j, m}(t): j=1, \ldots, N ; m=1, \ldots, N_{\perp}\right\} \\
\left\{\widehat{M}_{j, m}(t): j=1, \ldots, N ; m=1, \ldots, N_{\perp}\right\} \\
\left\{\widehat{E}_{0, j, m}(t): j=1, \ldots, N ; m=1, \ldots, N_{\perp}\right\} \\
\left\{\widehat{E}_{1, j, m}(t): j=1, \ldots, N ; m=1, \ldots, N_{\perp}\right\}
\end{array}\right) \equiv\left(\begin{array}{c}
\mathbf{u}_{1} \\
- \\
\mathbf{u}_{2}
\end{array}\right)
$$

denotes the vector of dependent variables, and $\mathbf{u}^{\prime}$ denotes the corresponding vector of time derivatives. We group $\mathbf{u}$ in (47) by acoustic variables $\mathbf{u}_{1}$ and light variables $\mathbf{u}_{2}$, but the specific ordering of the elements of the subvectors $\widehat{n}_{j, m}$, $\widehat{M}_{j, m}, \widehat{E}_{0, j, m}$ and $\widehat{E}_{1, j, m}$ is unimportant, so long as the ordering is consistent between the four subvectors of unknowns.

To advance numerically the system (46) from time $t_{n-1}$ to time $t_{n}$, we apply a variable-order, variable-step backward difference formula (BDF) as implemented in the differential-algebraic solver IDA [22]. For the purposes of our algorithm description, this discretization can be written for order $k$ in the classical linear multistep form ${ }^{2}$

$$
\mathbf{u}_{n}^{\prime}=\Delta t_{n}^{-1} \sum_{r=0}^{k} \alpha_{n, j} \mathbf{u}_{n-r}
$$

where $\mathbf{u}_{n}$ and $\mathbf{u}_{n}^{\prime}$ are approximations of $\mathbf{u}$ and $\mathbf{u}^{\prime}$ at time $t_{n}$, respectively, and the time step is $\Delta t_{n}=t_{n}-t_{n-1}$. The coefficients $\alpha_{n, r}$ depend only on the order $k$ and the history of the time steps, although in practice, the coefficients $\alpha_{n, r}$

$\overline{2}$ While the classical multistep formula is equivalent, in practice, the BDF algorithm is expressed typically in terms of predictor and corrector interpolary polynomials in time $[23,24]$. 
for $r=1,2, \ldots, k$ are never calculated explicitly [23,24]. Only the coefficient

$$
\alpha_{n, 0}=\sum_{r=1}^{k} \frac{\Delta t_{n}}{t_{n}-t_{n-r}}
$$

needs to be known explicitly outside of the approximation (48).

Insertion of the formula (48) in (46) results in a nonlinear algebraic system

$$
\mathbf{g}_{n} \equiv \mathbf{g}\left(\mathbf{u}_{n}\right) \equiv \mathbf{f}\left(t_{n}, \mathbf{u}_{n}, \Delta t_{n}^{-1} \sum_{r=0}^{k} \alpha_{n, r} \mathbf{u}_{n-r}\right)=\mathbf{0}
$$

to be solved at each time step. This nonlinear system (50) is solved using Newton iteration

$$
\mathbf{u}_{n}^{\ell+1}=\mathbf{u}_{n}^{\ell}+\delta \mathbf{u}_{n}^{\ell}, \quad \ell=0,1, \ldots
$$

where $\mathbf{u}_{n}^{\ell}$ is the $\ell^{t h}$ approximation of $\mathbf{u}_{n}, \delta \mathbf{u}_{n}^{\ell}$ is an approximate solution of the linear system

$$
\mathbf{J}\left(\mathbf{u}_{n}^{\ell}\right) \delta \mathbf{u}_{n}^{\ell}=-\mathbf{g}\left(\mathbf{u}_{n}^{\ell}\right)
$$

and $\mathbf{J}\left(\mathbf{u}_{n}^{\ell}\right)$ is the system Jacobian, i.e.,

$$
\mathbf{J}\left(\mathbf{u}_{n}\right) \equiv \frac{\partial \mathbf{g}_{n}}{\partial \mathbf{u}_{n}}=\frac{\partial \mathbf{f}_{n}}{\partial \mathbf{u}_{n}}+\frac{\partial \mathbf{u}_{n}^{\prime}}{\partial \mathbf{u}_{n}} \frac{\partial \mathbf{f}_{n}}{\partial \mathbf{u}_{n}^{\prime}}=\frac{\partial \mathbf{f}_{n}}{\partial \mathbf{u}_{n}}+\frac{\alpha_{n, 0}}{\Delta t_{n}} \frac{\partial \mathbf{f}_{n}}{\partial \mathbf{u}_{n}^{\prime}}
$$

evaluated at $\mathbf{u}_{n}^{\ell}$. Note that, in the Jacobian, only the coefficient $\alpha_{n, 0}$ appears explicitly, because

$$
\frac{\partial \mathbf{u}_{n-r}}{\partial \mathbf{u}_{n}}=0, \quad r=1,2, \ldots, k
$$

that is, the previous solution vectors are independent of the current solution vector. All of the $\alpha_{n, r}$ for $r=0,1, \ldots, k$ are implicitly involved in the computation of $\mathbf{g}\left(\mathbf{u}_{n}^{\ell}\right)$ for (52) since they are used to approximate $\mathbf{u}_{n}^{\prime}$ as in (50).

To describe in more detail the algorithm employed in evaluating the Newton 
update (51), we formally express the Jacobian as

$$
\mathbf{J}\left(\mathbf{u}_{n}^{\ell}\right) \equiv\left(\begin{array}{c|c}
\mathbf{J}_{11} & \mathbf{J}_{12} \\
\hline \mathbf{J}_{21} & \mathbf{J}_{22}
\end{array}\right)=\left(\begin{array}{cc|cc}
\mu \mathbf{I}-\mathbf{M} & -\mathbf{I} & \mathbf{0} & \mathbf{0} \\
-\mathbf{C} & \lambda \mathbf{I}-\mathbf{M} & -\rho \mathbf{E}_{1}^{*} & -\rho \mathbf{E}_{0}(\cdot)^{*} \\
\hline \sigma \mathbf{E}_{1} & \mathbf{0} & \mathbf{P} & \sigma \mathbf{N} \\
\sigma \mathbf{E}_{0}(\cdot)^{*} & \mathbf{0} & \sigma \mathbf{N}^{*} & \mathbf{P}
\end{array}\right),
$$

where the scalar coefficients are

$$
\mu \equiv \frac{1}{i \omega_{a}} \frac{\alpha_{n, 0}}{\Delta t_{n}}, \quad \lambda \equiv \mu_{n}+\frac{2 \nu_{a}}{i \omega_{a}}, \quad \rho \equiv \frac{Z m_{e}}{2 m c_{s}^{2}}\left(\frac{e}{m_{e} \omega}\right)^{2}, \quad \sigma \equiv \frac{i k}{4 \eta_{0} n_{c}} .
$$

The $N N_{\perp} \times N N_{\perp}$ sub-blocks in $\mathbf{J}$ are the identity matrix $\mathbf{I}$, the diagonal matrices

$$
\begin{aligned}
\mathbf{M} & \equiv \operatorname{diag}\left(M_{0, m}^{n}\right), \\
\mathbf{C} & \equiv \operatorname{diag}\left(\left(c_{0, m}^{n} / c_{s}\right)^{2}\right), \\
\mathbf{N} & \equiv \operatorname{diag}\left(\widehat{n}_{j, m}^{n, l}\right), \\
\mathbf{E}_{0} & \equiv \operatorname{diag}\left(\widehat{E}_{0, j, m}^{n, l}\right), \\
\mathbf{E}_{1} & \equiv \operatorname{diag}\left(\widehat{E}_{1, j, m}^{n, l}\right),
\end{aligned}
$$

and the discrete operator

$$
\mathbf{P} \equiv \mathbf{D}_{h_{x}}-\frac{i}{2 k_{0}} \boldsymbol{\Delta}_{h_{y}}+\nu_{c} \mathbf{I}+\frac{\sigma}{2} \operatorname{diag}\left(n_{0, m}^{n}-\bar{n}_{0}^{n}\right)
$$

where $\mathbf{D}_{h_{x}}$ and $\boldsymbol{\Delta}_{h_{y}}$ represent the discrete first derivative and Laplacian operators, respectively, corresponding to the spatial discretizations in (44) and (45). ${ }^{3}$ $\overline{3}$ To be more precise, for $j=1$, only the terms of $\mathbf{D}_{h_{x}}$ and $\boldsymbol{\Delta}_{h_{y}}$ for $j=1$ are included in $\mathbf{P}$. The remaining terms corresponding to initial data at $j=0$ are included on the right-hand side. 
The values of the indices $j$ and $m$ in (57) coincide with those of the elements of $\delta \mathbf{u}$ which they multiply, and the $(\cdot)^{*}$ notation is intended to indicate that, when $\mathbf{J}_{12}$ and $\mathbf{J}_{21}$ are multiplied times a vector, the factors preceding the $(\cdot)^{*}$ multiply the conjugate of the corresponding vector elements.

The linear system (52) for the Newton correction is itself solved iteratively during each Newton iteration step via a block Gauss-Seidel algorithm, which only requires the multiplication of $\mathbf{J}_{12}$ and $\mathbf{J}_{21}$ times vectors. Writing (52) in block form as

$$
\left(\begin{array}{ll}
\mathbf{J}_{11} & \mathbf{J}_{12} \\
\mathbf{J}_{21} & \mathbf{J}_{22}
\end{array}\right)\left(\begin{array}{l}
\delta \mathbf{u}_{n, 1}^{\ell} \\
\delta \mathbf{u}_{n, 2}^{\ell}
\end{array}\right)=-\left(\begin{array}{c}
\mathbf{g}_{n, 1}^{\ell} \\
\mathbf{g}_{n, 2}^{\ell}
\end{array}\right)
$$

we perform the iteration

$$
\begin{aligned}
& \delta \mathbf{u}_{n, 1}^{\ell, q+1}=-\mathbf{J}_{11}^{-1}\left(\mathbf{g}_{n, 1}^{\ell}+\mathbf{J}_{12} \delta \mathbf{u}_{n, 2}^{\ell, q}\right) \\
& \delta \mathbf{u}_{n, 2}^{\ell, q+1}=-\mathbf{J}_{22}^{-1}\left(\mathbf{g}_{n, 2}^{\ell}+\mathbf{J}_{21} \delta \mathbf{u}_{n, 1}^{\ell, q+1}\right)
\end{aligned}
$$

for $q=1,2, \ldots$, where, for example, $\mathbf{J}_{11}=\mathbf{J}_{11}\left(\mathbf{u}_{n}^{\ell}\right)$ and $\left(\delta \mathbf{u}_{n, 1}^{\ell, q}, \delta \mathbf{u}_{n, 2}^{\ell, q}\right)$ is the $q^{\text {th }}$ approximation of the Newton correction $\delta \mathbf{u}_{n}^{\ell}$. The application of $\mathbf{J}_{11}^{-1}$ in (59a) is a trivial operation, since the matrix $\mathbf{J}_{11}$ can be re-ordered as a block diagonal system with $2 \times 2$ blocks. The application of $\mathbf{J}_{22}^{-1}$ in (59b) is more complicated, however, since it requires the solution of the coupled-mode system (44)-(45) with a non-zero right-hand side. The coupled-mode system (44)-(45) is solved by a sweeping algorithm beginning at the left boundary and solving a sequence of linear systems for the planes of unknowns corresponding to successive values of $j$. These linear systems are solved using a block-Jacobi algorithm, where a tridiagonal linear system solver is used to invert the diagonal blocks.

Hence, the solution of the nonlinear system (50) at each time step via the 
algorithm just described involves three levels of nested iteration: the Newton iteration (51), the block-Gauss-Seidel iteration (59), and the block-Jacobi iterative solution of the linear systems solved in the sweeping algorithm used to apply $\mathbf{J}_{22}^{-1}$ in each step of $(59 \mathrm{~b})$. This raises the issue of sensible convergence tolerances for each of the iterative processes. In applying this algorithm to the problems described in the next section and others, we have observed that the convergence of the $\mathbf{J}_{22}^{-1}$ iteration is typically quite rapid, achieving several digits of accuracy in the relative residual in few iterations. Moreover, it is usually sufficient to perform only a single iteration of (59) to obtain rapid convergence of the Newton iteration, using the solution at the previous time step as the initial guess. Thus, the best general strategy is to perform only a small number of linear system iterations to minimize the cost of each Newton iteration. If at some point the number of Newton iterations required to reach the desired tolerance becomes unacceptably large, tighter tolerances on the inner linear iterations can be specified.

In our implementation of the preceding algorithm, we used the general-purpose solver IDA (Implicit Differential-Algebraic) [22], which is available as part of the SUNDIALS software suite from www.llnl.gov/CASC/sundials. IDA is an ANSI C, MPI-parallel implementation of an earlier FORTRAN code DASPK $[25,26]$ that supports automatic time step and integration order $k$ (up to $k=5$ ) adaption for the backward difference formula (48) based on userprovided error criteria. To advance the solution, IDA controlled the Newton update, computing $\mathbf{u}_{n}^{\ell}$ by (51) and $\left(\mathbf{u}_{n}^{\ell}\right)^{\prime}$ by (48). Taking $\mathbf{u}_{n}^{\ell},\left(\mathbf{u}_{n}^{\ell}\right)^{\prime}, \Delta t_{n}$, and $\alpha_{n, 0}$ as input from IDA, our routines implemented the inner two iterations that solve (59), subsequently providing to IDA the Newton corrections $\delta \mathbf{u}_{n}^{\ell}$ needed to form the update (51). 


\section{Numerical Results}

We now present some numerical examples to demonstrate the behavior of the energy transfer model in various regimes. We assume that the pump and probe beams have the same diameter $D \equiv D_{0}=D_{1}$ and consider a "narrow beam" problem where $D=10 \lambda$, a "medium beam" problem where $D=100 \lambda$, and a "wide beam" problem where $D=1000 \lambda$. For each case, we investigate the dependence of the energy transfer upon the initial velocity scale length over the range $L_{\perp}^{0} / D=1,5,10$ and 100 .

In all of the test cases, we consider a pair of $0.351 \mu \mathrm{m}$-wavelength beams crossing in a square domain $0 \leq x \leq L,-L / 2 \leq y \leq L / 2$, where we take $L=7.5 D$. The intensity profiles of the incident pump and probe beams at the left $(x=0)$ boundary are

$$
I_{b}(y)=I_{b}^{\max } \exp \left[-2\left(\frac{2\left|y-a_{b}\right|}{D}\right)^{6} \log (2)\right], \quad b=0,1,
$$

respectively. Here, $D$ denotes the full-width, half-maximum beam spot diameter; $a_{0}$ and $a_{1}$ are the $y$-coordinates of the pump and probe beam spot centers, respectively. Choosing $a_{0}=0.85 D$ and $a_{1}=-0.85 D$, we aim the beams at angles $\pm 12.5^{\circ}$ to the horizontal (i.e., , a $25^{\circ}$ angle between the beams) so as to intersect, in the absence of plasma, at the center of the domain. The maximum pump and probe intensities are specified as $I_{0}^{\max }=10^{15} \mathrm{~W} / \mathrm{cm}^{2}$ and $I_{1}^{\max }=1 \mathrm{~W} / \mathrm{cm}^{2}$, respectively. The use of a weak probe avoids pump depletion in all of the test cases, including the wide beam cases that achieve the largest gain. This allows more direct comparison with the analytic gain prediction, which assumes no pump depletion.

We assume that the background plasma evolves as the adiabatic rarefac- 
tion (33) about $y=0$ with $\gamma=5 / 3$ and $\alpha=0.1$. The initial electron and ion temperatures are $2.5 \mathrm{keV}$ and $0.5 \mathrm{keV}$, respectively. Using the speciesaveraged ionization $Z=3.5$ for a $C H$ plasma therefore yields the sound speed $c_{s}=4.76 \times 10^{5} \mathrm{~m} / \mathrm{sec}$, which in turn yields the acoustic frequency $\omega_{a}=$ $2 k_{\perp} c_{s}=3.69 \times 10^{12} \mathrm{rad} / \mathrm{sec}$. The damping rate is $\nu_{a}=0.1 \omega_{a}$, and inverse Bremsstrahlung absorption is neglected in these simulations. The model system is discretized as described in Section 3.2 on a spatial grid of size $10 D / \lambda \times$ $16 D / \lambda$, which is adequate to obtain several digits of accuracy in the probe gain. A simulation time of 50 picoseconds is used for all runs.

\subsection{Probe Gains}

The primary quantity of interest in these crossed beam calculations is the energy transfer between the beams. We characterize this by the probe gain

$$
G \equiv \frac{1}{2} \log \left(\frac{\int_{-L / 2}^{L / 2} I_{1}(L, y) d y}{\int_{-L / 2}^{L / 2} I_{1}(0, y) d y}\right),
$$

which was computed at the end of each time step. The integrals in (61) denote numerical quadratures of the probe intensity over the $z=0$ entrance plane and $z=L$ exit plane.

The gain (61) computed by the time-dependent coupled mode (TDCM) model is compared with the steady-state, analytic (SSA) model (38) where the integrals are evaluated numerically, including assumed Gaussian beam profiles (60). It is expected that the analytic model will over-predict the energy transfer because it fails to account for refraction and diffraction, both of which, in this geometry, tend to direct energy away from the region of strongest interaction. Of course the effects of refraction diminish with increasing velocity 
scale length, and so discrepancies at long scale lengths should primarily be due to diffraction.

In addition, a steady-state coupled mode (SSCM) model, where the coupled light equations (31c) and (31d) are solved using the steady state acoustic response (27), is evaluated. This third set of computations is included as an attempt to demonstrate the effect on energy transfer by the phenomena of refraction and diffraction and additionally isolates important time-dependent effects.

\subsubsection{Narrow beams}

[Fig. 4 about here.]

[Fig. 5 about here.]

We consider first a pair of beams with $D=10 \lambda$. In Figure 4, the probe gain $G$ computed by (61) is plotted as a function of time for four values of the initial velocity scale length: $L_{\perp}^{0}=D, L_{\perp}^{0}=5 D, L_{\perp}^{0}=10 D$, and $L_{\perp}^{0}=100 D$. The curves without circles denote the gain predicted by the TDCM code. The curves with circles denote the predictions obtained by the SSA model. Note that this steady-state model does in fact vary with time because it is evaluated at each time using the time-dependent velocity scale length $L_{\perp}$ given in (33).

In all four cases, the disagreement between the SSA and the TDCM gains at early time $(t<10 \mathrm{ps})$ occurs because the steady-state model cannot predict the transient growth of the acoustic wave, which concludes after roughly four damping times. Beyond $t=20$ ps or so, the agreement of the models is good, although the SSA results consistently over-predict the gain. In fact, the SSA 
curves for the $L_{\perp}^{0}=5 D$ and $L_{\perp}^{0}=10 D$ cases appear to diverge somewhat from the TDCM gains near 50 ps. In addition, for $t<20$ ps in the shortest scale length case, the SSA and TDCM models disagree substantially. Certainly refraction is the main suspect, as this case starts with severe beam bending and only a small portion of the interaction region initially overlaps with the sonic region. By 50 ps the background scale length is approximately $10 D$, so the beam bending is significantly reduced, and the agreement is better.

In Figure 5, the gain as computed using the SSCM code is plotted as curves with diamonds. There is excellent agreement in the long velocity scale length case where there is little refraction, which suggests that the discrepancy between the SSA gain and the TDCM result is due to diffraction. The intermediate SSCM cases are similarly closer to the TDCM curves at longer times suggesting that the curves are asymptotic to the same values. However, for the shortest scale length case, there is still substantial disagreement in the interval $10 \mathrm{ps} \lesssim t \lesssim 30 \mathrm{ps}$, which suggests that a reduced interaction region due to beam bending cannot be the lone cause. Surprisingly, the $L_{\perp}^{0}=5 D \mathrm{SSCM}$ gain is actually higher than the SSA result for this same time interval. The physics of the SSCM and TDCM codes differs only in the inclusion of time derivative terms, which suggests the presence of a time dependent mechanism that postpones the onset of a steady state and suppresses the gain.

\subsubsection{Medium beams}

[Fig. 6 about here.]

[Fig. 7 about here.] 
We next consider a pair of beams with $D=100 \lambda$. The length scales are all an order of magnitude bigger than for the narrow beam case, increasing the interaction length and thus the gain by roughly an order of magnitude. However, the time scale for the background flow $\left(L_{\perp}^{0} / c_{s}\right)$ is an order of magnitude slower because the reference sound speed is held fixed.

In Figure 6, the probe gains computed by the SSA and TDCM models are again plotted as a function of time for the four values of the initial velocity scale length. With the exception of the shortest scale length case, the two models agree well after the initial transient period. For the $L_{\perp}^{0}=D$ case, where refraction is initially quite strong, there is substantial disagreement over the entire 50 ps simulation. With the reduced time scale, the velocity scale length in this case changes by only a factor of two over a 50 ps run and so significant refraction occurs for the duration.

The probe gain computed by the SSCM model is presented in Figure 7. There is better agreement between the SSCM and TDCM models as compared to the SSA and TDCM models for the two longer scale length cases. Nevertheless, a sizable discrepancy in the shortest scale length case is evident, and as before, the gain in the $L_{\perp}^{0}=5 D$ case as computed by SSCM is higher than the SSA result. Again this points to a significant time-dependent effect causing gain suppression that has stronger effects as the scale length decreases.

\subsubsection{Wide beams}

[Fig. 8 about here.]

[Fig. 9 about here.] 
Finally, we consider a pair of beams with $D=1000 \lambda$. Relative to the medium beam case, the length scales are increased by another order of magnitude and the background time scale reduced by another order of magnitude. The gain should be roughly one order of magnitude higher.

We note that these wide beam calculations are very large, since a $10000 \times$ 16000 spatial grid was required, yielding a total of 160 million mesh cells, with four complex dependent variables per cell. Approximately 7.5 to 16 wall-clock hours on 400 processors of the MCR Intel GNU/Linux cluster were required to complete 50 ps of simulation time, with the runtime increasing with decreasing scale length. In comparison, the 25 zone numerical quadrature of the SSA model, computed at ten intervals of five picoseconds each in a MATLAB script, took approximately 4 seconds on a single processor of the same machine.

In Figure 8, the probe gains computed by the SSA and TDCM models are again plotted as a function of time for the four values of the initial velocity scale length. As before good agreement between the models is seen, except in the shortest scale length case. In Figure 9, excellent agreement between the SSCM and TDCM models is demonstrated, with the now familiar exceptions of the shorter scale length cases. The nature of the implicated time-dependent effects will be identified in the next section.

\subsection{Beam Motion and Frequency Mismatch}

In all short scale-length cases $\left(L_{\perp}^{0}=D\right)$, the gain computed by the TDCM code is less than that predicted by either of the steady-state methods. This suggests that a time-dependent mechanism exists in the short scale length case 
that postpones the achievement of steady-state. In the short scale length case, the refraction is not only strong, but the time-dependence of the background flow alters the refraction significantly, that is, the beams move at a significant fraction of the sound speed. As discussed in Section 2.1, this beam motion is believed to be a mechanism that detunes the three-wave resonance.

We first will demonstrate empirically that beam motion is closely correlated with gain suppression. Direct consideration of the magnitude of the transverse beam velocities provides further support for this explanation. By postprocessing the coupled-mode calculations, the validity of the phase-shifted formula (29) will be demonstrated. Finally, the phase mismatch will be shown to correspond to a shift of the resonant region, an effect that has significance for the proper interpretation of cross-beam experiments in unsteady plasma flows.

[Fig. 10 about here.]

As the phenomenon occurs for all beam widths, just at different rates, without loss of generality, we consider the medium beam, short initial scale length case. We conduct an experiment where the background flow is held fixed until $t_{c}=$ 20 picoseconds, after which it is allowed to resume its normal time variation, that is,

$$
L_{\perp}= \begin{cases}L_{\perp}^{0}+\frac{\gamma+1}{2} c_{s} t_{c}, & \text { for } 0 \leq t \leq t_{c} \\ L_{\perp}^{0}+\frac{\gamma+1}{2} c_{s} t, & \text { for } t \geq t_{c} .\end{cases}
$$

Thus, the background is initially steady, and while there is refraction, there is no beam motion. At $20 \mathrm{ps}$, the background begins to move impulsively causing the beams to move. The TDCM gain resulting from this experiment is plotted in Figure 10 against the steady-state SSCM gain and the TDCM 
gain computed with the unaltered background flow. After the transient, a time suitable for those acoustic waves no longer ponderomotively forced to damp, the gain curve for this experiment comes into agreement with with the unaltered TDCM gain curve. This points to beam motion as the cause for the drop in gain.

Note that, since the beam refraction is computed as an instantaneous quantity given the imposed background flow, there is no memory effect inherent in the beam motion. The damping of the acoustic waves erases memory of past forcing, and so after several damping times, there is no lasting dependence on the path of evolution. Indeed, Figure 10 demonstrates this, since the two TDCM gain curves quickly come into agreement after the history of the acoustic waves is damped out.

How does the beam motion alter the gain and why does it only do so in the shortest scale length case? While the ponderomotive forcing is relatively steady in the frame attached to the intersection of the beams, the refractioninduced translation of the interaction region relative to the lab frame creates an effective frequency for the forcing. Specifically, a sinusoidal forcing pattern in $y$ with wavenumber $2 k_{\perp}$ translating relative to a fixed point with velocity $v$ appears as a frequency of $2 k_{\perp} v$ in the fixed frame. Of course, this is a crude argument since both the fluid and the ponderomotive forcing are accelerating (decelerating) relative to laboratory frame. Nevertheless, we assert that the phase mismatch $\partial_{t} \phi_{a}$ in (29) is approximately this frequency, $\dot{\phi}_{a}(t) \approx 2 k_{\perp} v(t)$. A similar argument can be made associating $\partial_{t}^{2} \phi_{a}$ with the deceleration of ponderomotive force. The requirement for phase mismatch terms to be important 
is that they compete with the damping term, for example,

$$
\frac{\dot{\phi}_{a}(t)}{\omega_{a}} \gtrsim \frac{\nu_{a}}{\omega_{a}} \Rightarrow \frac{v}{c_{s}} \gtrsim \frac{\nu_{a}}{\omega_{a}} .
$$

This relative damping rate is one tenth for the plasma considered here, so transverse velocities of at least ten percent of the sound speed are required.

[Fig. 11 about here.]

In Figure 11, estimates of the transverse velocity of the interaction region are plotted. These are obtained by integrating the ray equations for the rarefaction density profile and computing the velocity of the intersection of the central ray of each beam. In the isothermal case, an exact form for the ray trajectory and its time derivative can be derived (See Appendix E), but for the adiabatic equations, the system must be integrated numerically.

Note that the transverse velocity of the ray intersection point as a fraction of the sonic line sound speed has the same evolution for each of the three beam widths when the time is scaled by the beam diameter. From the figure it is obvious that for the shortest scale length problem, $L_{\perp}^{0}=D$, the transverse velocity is initially of the same order of magnitude of $\nu_{a} c_{s} / \omega_{a}$. With increasing scale length, the transverse velocity rapidly diminishes, due to the fact that the density gradient is reduced as the velocity scale length increases. That the behavior of the deceleration of the interaction region is very similar can be ascertained by considering the slopes of the curves. We therefore would expect to see the most dramatic effects of frequency mismatch in the shortest scale length case and perhaps somewhat in the $L_{\perp}^{0}=5 D$ case. Indeed this was shown in Section 4.1.

Compelling a posteriori evidence of the effects of frequency mismatch can be 
obtained in a post-processed consistency check by evaluating the gain from the analytic acoustics responses, that is, equations (27) and (29). This is done as follows:

(1) The time-dependent, self-consistent electric and acoustic fields at some time are obtained from the TDCM code.

(2) The gain is computed using the steady-state density perturbations (27):

(a) the acoustic response is computed from the steady-state formula (27) using the TDCM electric fields;

(b) the electric fields are recomputed using this assumed density perturbation;

(c) and the gain is computed from these new electric fields.

(3) The gain is computed using the steady-state density perturbations with frequency mismatch (29):

(a) the acoustic response is computed from the steady-state formula with frequency mismatch (29) using the TDCM electric fields and frequency shifts approximated from the TDCM density perturbation;

(b) the electric fields are recomputed using this assumed density perturbation;

(c) and the gain is computed from these new electric fields.

Step 3(a) involves computing a local estimate of the density perturbation frequency and its time derivative. Noting that, for $\widehat{n}=|\widehat{n}| \exp i \phi(t)$,

$$
\frac{\partial_{t} \widehat{n}}{\widehat{n}}=i \partial_{t} \phi+\frac{\partial_{t}|\widehat{n}|}{|\widehat{n}|}, \quad(|\widehat{n}|>0)
$$

We make the approximation

$$
\partial_{t} \phi_{a} \approx \partial_{t} \phi=\operatorname{Im}\left\{\frac{\partial_{t} \widehat{n}}{\widehat{n}}\right\}
$$


which is reasonable after the initial start-up transients have damped away. The time derivative of $\partial_{t} \phi_{a}$ is approximated using first-order finite differencing.

[Fig. 12 about here.]

The results of these post-processed gain predictions are plotted in Figure 12. In comparison with the directly computed TDCM result, the gain calculated using the original steady-state density formula (27) is over-predicted, as expected. The gain calculated using the modified steady-state density formula that accounts for frequency mismatch (29), after an initial layer where the approximation (65) is dubious, is in excellent agreement with the directly computed TDCM gain and strongly supports the frequency mismatch theory.

Finally, careful consideration of (29) suggests that the frequency mismatch can be interpreted as a shift of the resonant region. Without any shift, resonance occurs where the real part of the denominator vanishes and is limited by the small non-zero damping. In the presence of a frequency mismatch, the entire denominator could potentially vanish, but we restrict our consideration only to the case where the real part of the denominator vanishes. Choosing the root closest to $y=0$, this occurs at

$$
\frac{y}{L_{\perp}}=\frac{2}{\gamma+1} \frac{\partial_{t} \phi_{a}}{\omega_{a}}
$$

For $0.1 \leq\left|\partial_{t} \phi_{a} / \omega_{a}\right| \leq 1$, this gives an effective shift of $3-30 \mu \mathrm{m}$ for the short scale length, medium beam width case. Associating the frequency mismatch with the downward translation of the ponderomotive forcing suggests that $\partial_{t} \phi_{a} \leq 0$, which corresponds to a shift into regions of slower plasma velocity. This could be an important effect in experiments where beams are aimed to intersect or avoid a resonance region. 
[Fig. 13 about here.]

As evidence of a downward shift of the resonance region in the $L_{\perp}^{0}=D$ case, consider a sequence of such problems in which the pair of beams is also shifted downward by varying amounts. As before, we fix the background flow for the first $20 \mathrm{ps}$ and then let it vary normally. In Figure 13, the probe gain is plotted for the original, unshifted beams (dotted lines), a 10-micron downward shift (dash-dot line), a 20-micron downward shift (solid line), and a 30-micron downward shift (dashed line). All four cases reach a steady-state gain before the 20 ps cutoff. At the cutoff, when the background plasma is again permitted to evolve, all four curves exhibit a transient response lasting for a few picoseconds, which is approximately the characteristic damping time $\nu_{a}^{-1}$. After this transient, the gain curves for the unshifted and 10-micron cases are significantly lower than during the pre-cutoff interval, and the jump in the 10-micron shifted curve is somewhat less than in the unshifted case. The 20-micron shift curve begins to increase from about its pre-cutoff value, whereas the gain for the 30-micron shifted beams increases dramatically after the cutoff.

The behavior of the gain curves in Figure 13 is consistent with the hypothesis that, prior to the cutoff, the resonance region is centered on the sonic line because there is no beam motion, while after the cutoff the resonance region has shifted downward as much as nearly 20 microns. The size of the resonance shift will differ for each case because the background gradient, and thus the transverse beam velocities change. For the unshifted and 10-micron shifted curves, the behavior is consistent with a sudden downward shift of the resonance region at the cutoff time the moves the resonance region further away from the beam interaction region, decreasing the gains. In the 20-micron shift 
case, the gain is relatively unchanged, which is consistent with a shift of the resonant region such that it remains within the interaction region. When the beam pair is shifted by 30 microns, the hypothesized downward shift of the resonance region at the cutoff apparently results in a larger overlap of the interaction region than before the cutoff, with a corresponding gain increase.

\section{Conclusions}

Crossed laser beams in an expanding flow can exchange energy through a beatwave-driven ion acoustic wave. To predict the behavior, we have derived a nonlinear, coupled-mode model of this process composed of a pair of temporal ordinary differential equations describing the ion acoustic response and a pair of paraxial light equations. Restricting the light model to two dimensions, the numerical discretization of the coupled mode system of differential-algebraic equations was presented in detail. To interpret the probe beam gain predictions, the amplitude gains from this numerical model were compared with gains integrated numerically from a steady-state acoustic response evaluated using ray-traced beam positions.

A series of numerical experiments for varying beam diameters were presented to demonstrate the effects of unsteady beam refraction on probe gain. These numerical simulations were based on a self-similar background flow characterized by an initial velocity length scale $L_{\perp}$, and the probe beam intensity was purposely chosen to be small relative to the pump beam intensity to eliminate the effects of nonlinear pump depletion in the comparisons. It was found that when $L_{\perp}$ was large compared to the beam diameters, the models produced similar results and discrepancies were attributable to beam diffraction. How- 
ever, when $L_{\perp}$ was the same order as the beam diameters, unsteady effects in the beam propagation lead to reduced energy transfer between the beams, and thus a large discrepancy between the steady-state and unsteady models. This gain suppression was attributed to a phase shift detuning the three-wave resonance and interpreted as an effective shift of the resonant region in the flow. As such, the possible occurrence and effects of unsteady beam refraction should be considered in the design and analysis of crossed-beam experiments with steep initial flow gradients and significant flow evolution.

One-dimensional coupled mode models are often used to approximate and understand laser plasma interactions. In such investigations, the background plasma is assumed frequently to be uniform. The method described here demonstrates a numerical technique to address nonlinear coupled mode models in two and, by extension, three dimensions with prescribed transverse plasma gradients. Indeed, the obvious extension of the current work is to replace the analytically prescribed background flow with experimentally measured backgrounds or backgrounds computed using fully nonlinear hydrodynamic models. Given the constraints on computational resources, it is unlikely that any computational tool including all of the physical plasma and laser interaction effects will be available in the near term for rapid experimental design and analysis. Thus, reduced numerical models like the one presented here can be valuable tools to design and to understand future laser plasma experiments. 


\section{A Sound Speed Perturbations}

We begin with the differential relation (4). Further defining

$$
c_{a}^{2}=\left(c_{0}+\delta c\right)^{2}=c_{0}^{2}+2 c_{0} \delta c+\delta c^{2},
$$

substitution of (9) into (4) gives

$$
\left(d p_{0}-m c_{0}^{2} d n_{0}\right)+\left(d(\delta p)-m c_{0}^{2} d(\delta n)-2 m c_{0} \delta c d n_{0}\right)+\cdots=0
$$

where products of small terms have been omitted. Collecting terms of like order and setting each result to zero gives

$$
\begin{aligned}
d p_{0} & \approx m c_{0}^{2} d n_{0} \\
d(\delta p) & \approx m c_{0}^{2} d(\delta n)+2 m c_{0} \delta c d n_{0}
\end{aligned}
$$

However, by the definition of the $\gamma$-law sound speed,

$$
\begin{aligned}
c^{2} & =\frac{\gamma p}{m n}, \\
c_{0}^{2}\left(1+\frac{2 \delta c}{c_{0}}+\frac{\delta c^{2}}{c_{0}^{2}}\right) & =\frac{\gamma p_{0}}{m n_{0}}\left(1+\left[\frac{\delta p}{p_{0}}-\frac{\delta n}{n_{0}}\right]-\frac{\delta p}{p_{0}} \frac{\delta n}{n_{0}}+\cdots\right) .
\end{aligned}
$$

Again equating terms of like order, we find

$$
c_{0}^{2} \approx \frac{\gamma p_{0}}{m n_{0}} \quad \text { and } \quad \frac{2 \delta c}{c_{0}} \approx\left[\frac{\delta p}{p_{0}}-\frac{\delta n}{n_{0}}\right]
$$

Substituting these into (A.3b),

$$
d(\delta p) \approx m c_{0}^{2} d(\delta n)+m c_{0}^{2}\left[\frac{\delta p}{p_{0}}-\frac{\delta n}{n_{0}}\right] d n_{0}
$$

Rewriting in terms of $\delta p / p_{0}$ and $\delta n / n_{0}$,

$$
p_{0} d\left(\frac{\delta p}{p_{0}}\right)+\frac{\delta p}{p_{0}} d p_{0} \approx m n_{0} c_{0}^{2} d\left(\frac{\delta n}{n_{0}}\right)+m c_{0}^{2}\left(\frac{\delta n}{n_{0}}\right) d n_{0}+m c_{0}^{2}\left[\frac{\delta p}{p_{0}}-\frac{\delta n}{n_{0}}\right] d n_{0},
$$


and finally, substituting in (A.3a),

$$
d\left(\frac{\delta p}{p_{0}}\right) \approx \gamma d\left(\frac{\delta n}{n_{0}}\right) .
$$

We now integrate to find that

$$
\delta p \approx m c_{0}^{2} \delta n,
$$

where the constant of integration has been set to zero, i.e., , there are no density perturbations without corresponding pressure perturbations.

\section{B Coordinate Transformation}

It is convenient to consider the interaction region in the coordinate system aligned with the probe beam, $(\zeta, \xi)$. Note the following transformations:

$$
\begin{aligned}
& x=\zeta \cos \theta-\xi \sin \theta, \\
& y=\zeta \sin \theta+\xi \cos \theta, \\
& \zeta=x \cos \theta+y \sin \theta, \\
& \xi=-x \sin \theta+y \cos \theta .
\end{aligned}
$$

Furthermore,

$$
\begin{aligned}
& \left.\frac{\partial}{\partial x}\right|_{y}=\left.\left.\frac{\partial \zeta}{\partial x}\right|_{y} \frac{\partial}{\partial \zeta}\right|_{\xi}+\left.\left.\frac{\partial \xi}{\partial x}\right|_{y} \frac{\partial}{\partial \xi}\right|_{\zeta}=\left.\cos \theta \frac{\partial}{\partial \zeta}\right|_{\xi}-\left.\sin \theta \frac{\partial}{\partial \xi}\right|_{\zeta}, \\
& \left.\frac{\partial}{\partial y}\right|_{x}=\left.\left.\frac{\partial \zeta}{\partial y}\right|_{x} \frac{\partial}{\partial \zeta}\right|_{\xi}+\left.\left.\frac{\partial \xi}{\partial y}\right|_{x} \frac{\partial}{\partial \xi}\right|_{\zeta}=\left.\sin \theta \frac{\partial}{\partial \zeta}\right|_{\xi}+\left.\cos \theta \frac{\partial}{\partial \xi}\right|_{\zeta} .
\end{aligned}
$$

Thus, along a line of constant $\xi$,

$$
\left.\cos \theta \frac{\partial}{\partial x}\right|_{y}+\left.\sin \theta \frac{\partial}{\partial y}\right|_{x}+\left.\nu_{c} \equiv \frac{\partial}{\partial \zeta}\right|_{\xi}+\nu_{c}
$$




\section{Determination of the Integration Region}

We address the limits of integration, working with dimensional quantities for clarity. We assume symmetry and neglect refraction, so the region of interaction, as shown in Figure 3, is the diamond abcd with diagonals aligned with

the $(x, y)$ axes. By $(37)$, for a given $\xi$, we seek to integrate from edge $a b$ to edge $b c$ to obtain the amplitude of the probe along that $\xi$-ray after the integration. The equations for lines $\zeta_{0}(\xi)$ and $\zeta_{1}(\xi)$, which define the beginning and end of the interaction region, respectively, can be obtained from simple geometry.

The length of the side of the diamond $D_{d}$ is found from the relation between the sine of an angle and the legs and hypotenuse of a right triangle:

$$
D=D_{d} \sin 2 \theta \quad \Rightarrow \quad D_{d}=\frac{D}{\sin 2 \theta}
$$

The length of the longer diagonal of the diamond $d_{L}$ is obtained from the law of cosines,

$$
\begin{aligned}
d_{L} & =\sqrt{2 D_{d}^{2}[1-\cos (\pi-2 \theta)]} \\
& =D \sqrt{\frac{[1+\cos 2 \theta]}{2 \sin ^{2} \theta \cos ^{2} \theta}}, \\
& =D \csc \theta
\end{aligned}
$$

Similarly, the shorter diagonal $d_{S}$ is

$$
\begin{aligned}
d_{S} & =\sqrt{2 D_{d}^{2}[1-\cos (2 \theta)]}, \\
& =D \sqrt{\frac{1-\cos 2 \theta}{2 \sin ^{2} \theta \cos ^{2} \theta}}, \\
& =D \sec \theta
\end{aligned}
$$

Since both coordinate systems are collocated with the center of the diamond, we can easily determine the coordinates of each vertex in either frame, as 
listed in Table 1.

[Table 1 about here.]

Each pair of points uniquely determines a line. The $\zeta$-coordinate of lower limit in terms of $\xi$ is

$$
\frac{\zeta_{0}+\frac{1}{2} D \tan \theta}{\xi+\frac{1}{2} D}=\frac{-\cot \theta+\tan \theta}{2} \Rightarrow \zeta_{0}(\xi)=-\frac{2 \xi \cos 2 \theta+D}{2 \sin 2 \theta} .
$$

Similarly, the upper limit is

$$
\frac{\zeta_{1}-\frac{1}{2} D \cot \theta}{\xi+\frac{1}{2} D}=\frac{\tan \theta-\cot \theta}{2} \Rightarrow \zeta_{1}(\xi)=-\frac{2 \xi \cos 2 \theta-D}{2 \sin 2 \theta} .
$$

\section{Integration of the Exponent}

Returning to (37), for a given $\xi$, the gain across the interaction region is given by (38). We seek to determine the functional form of the integral in the exponent. We note that $M_{0}=M_{0}(\zeta, \xi)$ and $c_{0}=c_{0}(\zeta, \xi)$ and neglect $\zeta$-variation in $\widetilde{E}_{0}$ such that

$$
I(\xi) \equiv \int_{\zeta_{0}(\xi)}^{\zeta_{1}(\xi)} \Gamma\left(\zeta^{\prime}, \xi\right) d \zeta^{\prime}=\int_{\zeta_{0}(\xi)}^{\zeta_{1}(\xi)}\left[\frac{2 \nu_{a}}{\omega_{a}} M_{0}-i\left(M_{0}^{2}-\frac{c_{0}^{2}}{c_{s}^{2}}\right)\right]^{-1} d \zeta^{\prime} .
$$

Make the change of variables

$$
X=\frac{y}{L_{\perp}}=\left[\frac{\sin \theta}{L_{\perp}} \zeta^{\prime}+\frac{\cos \theta}{L_{\perp}} \xi\right] \Rightarrow d X=\frac{\sin \theta}{L_{\perp}} d \zeta^{\prime} .
$$

Then

$$
I(\xi)=\frac{L_{\perp}}{\sin \theta} \int_{X_{0}(\xi)}^{X_{1}(\xi)}\left[\frac{2 \nu_{a}}{\omega_{a}} M_{0}(X, \xi)-i\left(M_{0}^{2}(X, \xi)-\frac{c_{0}^{2}}{c_{s}^{2}}(X, \xi)\right)\right]^{-1} d X .
$$

Without loss of generality, we substitute the adiabatic background expansion (33) into (D.3); the isothermal result can be recovered by taking $\gamma=1$ 
in the result. Making this substitution, we obtain

$$
I(\xi)=\frac{L_{\perp}}{\sin \theta} \int_{X_{0}(\xi)}^{X_{1}(\xi)}\left[\frac{2 \nu_{a}}{\omega_{a}}(X+1)-i X\left(\left[1-\left(\frac{\gamma-1}{2}\right)^{2}\right] X+(\gamma+1)\right)\right]^{-1} d X
$$

Defining

$$
f(X) \equiv\left[1-\left(\frac{\gamma-1}{2}\right)^{2}\right] X+\frac{\gamma+1}{2}
$$

and

$$
\psi \equiv\left[\left(\frac{\gamma+1}{2}\right)^{2}-\left(\frac{\nu_{a}}{\omega_{a}}\right)^{2}+i \frac{\left(\gamma^{2}-1\right)}{2} \frac{\nu_{a}}{\omega_{a}}\right]^{-1 / 2},
$$

the solution is

$$
I(\xi)=\left.\frac{L_{\perp}}{\sin \theta} \psi \arctan \left[\psi\left\{\frac{\nu_{a}}{\omega_{a}}-i f(X)\right\}\right]\right|_{X_{0}(\xi)} ^{X_{1}(\xi)}
$$

To determine the real part of (D.7), first note that, for $x, y \in \mathbb{R}$,

$$
\begin{gathered}
\arctan [x+i y]=\frac{1}{2} \arctan \left[\frac{2 x}{1-x^{2}-y^{2}}\right]+i \frac{1}{4} \ln \left[\frac{x^{2}+(y+1)^{2}}{x^{2}+(y-1)^{2}}\right]+n \pi, \\
\sqrt{x+i y}= \pm \frac{\sqrt{2}}{2}\left(\sqrt{\sqrt{x^{2}+y^{2}}+x}+i \sqrt{\sqrt{x^{2}+y^{2}}-x}\right)
\end{gathered}
$$

where $n$ is any integer. Thus,

$$
\begin{aligned}
\psi & \equiv \psi_{r}+i \psi_{i} \\
& =\frac{\sqrt{2}}{2 r} \sqrt{r+\left(\frac{\gamma+1}{2}\right)^{2}-\left(\frac{\nu_{a}}{\omega_{a}}\right)^{2}}-i \frac{\sqrt{2}}{2 r} \sqrt{r-\left(\frac{\gamma+1}{2}\right)^{2}+\left(\frac{\nu_{a}}{\omega_{a}}\right)^{2}}
\end{aligned}
$$

where

$$
r^{2} \equiv\left[\left(\frac{\gamma+1}{2}\right)^{2}-\left(\frac{\nu_{a}}{\omega_{a}}\right)^{2}\right]^{2}+\left[\frac{\nu_{a}}{\omega_{a}} \frac{\left(\gamma^{2}-1\right)}{2}\right]^{2}
$$

and so

$$
\begin{aligned}
I(\xi)=\frac{L_{\perp}}{\sin \theta}\left(\psi_{r}+i \psi_{i}\right) \arctan \left[\left(\psi_{r} \frac{\nu_{a}}{\omega_{a}}\right.\right. & \left.+\psi_{i} f(X)\right) \\
& \left.+i\left(\psi_{i} \frac{\nu_{a}}{\omega_{a}}-\psi_{r} f(X)\right)\right]\left.\right|_{X_{0}(\xi)} ^{X_{1}(\xi)}
\end{aligned}
$$


Therefore,

$$
\begin{aligned}
& \operatorname{Re}\{I(\xi)\}= \\
& \quad \frac{L_{\perp}}{2 \sin \theta}\left(\psi_{r} \arctan \left[\frac{2\left(\psi_{r} \nu_{a} / \omega_{a}+\psi_{i} f(X)\right)}{1-\left(\psi_{r} \nu_{a} / \omega_{a}+\psi_{i} f(X)\right)^{2}-\left(\psi_{i} \nu_{a} / \omega_{a}-\psi_{r} f(X)\right)^{2}}\right]\right. \\
& \left.-\frac{\psi_{i}}{2} \ln \left[\frac{\left(\psi_{r} \nu_{a} / \omega_{a}+\psi_{i} f(X)\right)^{2}+\left(\psi_{i} \nu_{a} / \omega_{a}-\psi_{r} f(X)+1\right)^{2}}{\left(\psi_{r} \nu_{a} / \omega_{a}+\psi_{i} f(X)\right)^{2}+\left(\psi_{i} \nu_{a} / \omega_{a}-\psi_{r} f(X)-1\right)^{2}}\right]\right)\left.\right|_{X_{0}(\xi)} ^{X_{1}(\xi)} \cdot
\end{aligned}
$$

For an isothermal plasma, $\psi_{i}=0$, and the real component of the integral reduces to

$$
\begin{aligned}
& \operatorname{Re}\{I(\xi)\}=\left.\frac{L_{\perp}}{2 \sin \theta} \psi_{r} \arctan \left[\frac{2 \psi_{r} \nu_{a} / \omega_{a}}{1-\left(\psi_{r} \nu_{a} / \omega_{a}\right)^{2}-\left(\psi_{r} f(X)\right)^{2}}\right]\right|_{X_{0}(\xi)} ^{X_{1}(\xi)}, \\
&=\left.\frac{L_{\perp}}{2 \sin \theta} \psi_{r} \arctan \left[\psi_{r} \frac{\left(\nu_{a} / \omega_{a}\right)^{2}+X(1+X / 2)}{\nu_{a} / \omega_{a}}\right]\right|_{X_{0}(\xi)} ^{X_{1}(\xi)}, \\
&= \frac{L_{\perp}}{2 \sin \theta} \psi_{r}\left(\arctan \left[\psi_{r} \frac{\left(\nu_{a} / \omega_{a}\right)^{2}+X_{1}\left(1+X_{1} / 2\right)}{\nu_{a} / \omega_{a}}\right]\right. \\
&\left.-\arctan \left[\psi_{r} \frac{\left(\nu_{a} / \omega_{a}\right)^{2}+X_{0}\left(1+X_{0} / 2\right)}{\nu_{a} / \omega_{a}}\right]\right),
\end{aligned}
$$

where $\psi_{r}^{-1}=\sqrt{1-\left(\nu_{a} / \omega_{a}\right)^{2}}$. We note that

$$
\begin{aligned}
& X_{0}=\frac{\zeta_{0} \sin \theta+\xi \cos \theta}{L_{\perp}}=\frac{\xi-D / 2}{2 L_{\perp} \cos \theta}, \\
& X_{1}=\frac{\zeta_{1} \sin \theta+\xi \cos \theta}{L_{\perp}}=\frac{\xi+D / 2}{2 L_{\perp} \cos \theta} .
\end{aligned}
$$

\section{E Isothermal Ray Traced Solution}

Given the one-dimensional, self-similar rarefaction flow (33), a ray-traced model can approximate the beam paths and the motion of the interaction region. The two-dimensional ray-traced equations for propagation through a 
flow in the $y$-direction reduce to

$$
\begin{array}{ll}
\left.\frac{d y}{d x}\right|_{t}=\frac{q}{\cos \theta_{0}}, & y\left(x_{0}, t\right)=y_{0} \\
\left.\frac{d q}{d x}\right|_{t}=\left.\frac{1}{2 \cos \theta_{0}} \frac{\partial \eta^{2}}{\partial y}\right|_{t}, & q\left(x_{0}, t\right)=\sin \theta_{0}
\end{array}
$$

where $y(x, t)$ is the ray height at horizontal location $x$ and time $t, q$ is proportional to the normal to the wavefront, $\left(x_{0}, y_{0}\right)$ is the initial location of the ray at angle $\theta_{0}$ from the horizontal, and $\eta$ is the index of refraction given by

$$
\eta^{2}=1-\frac{n_{0}(y, t)}{n_{c}}
$$

For an adiabatic flow, the ray-tracing equations (E.1) must be integrated numerically as a system of ordinary differential equations. However, in the isothermal case,

$$
\eta^{2}=1-\alpha \exp \left(-\frac{y}{L_{\perp}}\right)
$$

and the ray-tracing equations (E.1) can be solved analytically.

Differentiating (E.1a) with respect to $x$ and substituting in (E.1b),

$$
\begin{gathered}
\left.\frac{\partial^{2} y}{\partial x^{2}}\right|_{t}=\left.\frac{1}{2 \cos ^{2} \theta_{0}} \frac{\partial \eta^{2}}{\partial y}\right|_{t}, \\
y\left(x_{0}, t\right)=y_{0},\left.\quad \frac{\partial y}{\partial x}\right|_{t}\left(x_{0}, t\right)=\tan \theta_{0} .
\end{gathered}
$$

Define

$$
\left.\left.u(y, t) \equiv \frac{\partial y}{\partial x}\right|_{t} \quad \Rightarrow \quad \frac{\partial^{2} y}{\partial x^{2}}\right|_{t}=\left.\frac{\partial u}{\partial x}\right|_{t}=\left.\left.\frac{\partial x}{\partial y}\right|_{t} \frac{\partial y}{\partial x}\right|_{t}=\left.\frac{1}{2} \frac{\partial u^{2}}{\partial y}\right|_{t} .
$$

Substituting this into (E.4a), we have

$$
\left.\frac{\partial u^{2}}{\partial y}\right|_{t}=\left.\frac{1}{\cos ^{2} \theta_{0}} \frac{\partial \eta^{2}}{\partial y}\right|_{t}
$$

Integrating gives

$$
u(y, t)= \pm \sqrt{u_{0}^{2}+\frac{\left(\eta^{2}-\eta_{0}^{2}\right)}{\cos ^{2} \theta_{0}}}
$$


where $u_{0}=u\left(y_{0}, t\right)$ and $\eta_{0}=\eta\left(y_{0}, t\right)$. Rewriting this in terms of $y$,

$$
\begin{aligned}
\left.\frac{\partial y}{\partial x}\right|_{t} & = \pm \sqrt{\tan ^{2} \theta_{0}+\frac{\alpha}{\cos ^{2} \theta_{0}}\left[\exp \left(-\frac{y_{0}}{L_{\perp}}\right)-\exp \left(-\frac{y}{L_{\perp}}\right)\right]} \\
& = \pm \sqrt{b-a \exp \left(-\frac{y}{L_{\perp}}\right)}
\end{aligned}
$$

where

$$
a \equiv \frac{\alpha}{\cos ^{2} \theta_{0}} \geq 0 \quad \text { and } \quad b \equiv \tan ^{2} \theta_{0}+a \exp \left(-\frac{y_{0}}{L_{\perp}}\right) \geq 0
$$

The positive root of (E.8) is the unique solution that satisfies the boundary condition at $\left(x_{0}, y_{0}\right)$.

Let $y=-L_{\perp} \ln z$, then $(\mathrm{E} .8 \mathrm{~b})$ can be written as

$$
\left.\frac{1}{z \sqrt{b-a z}} \frac{\partial z}{\partial x}\right|_{t}=-\frac{1}{L_{\perp}},
$$

where the right-hand side is independent of $x$. Integrating,

$$
\frac{2}{\sqrt{b}} \operatorname{arctanh}\left[\sqrt{1-\frac{a z}{b}}\right],=\frac{x+C}{L_{\perp}},
$$

where the constant of integration is

$$
C=-x_{0}+\frac{2 L_{\perp}}{\sqrt{b}} \operatorname{arctanh}\left[\frac{\tan \theta_{0}}{\sqrt{b}}\right] .
$$

Solving (E.11) for $y$, we arrive at

$$
y=-L_{\perp} \ln \left[\frac{b}{a} \operatorname{sech}^{2} \zeta\right]
$$

where

$$
\zeta \equiv \frac{\sqrt{b}}{2 L_{\perp}}\left(x-x_{0}\right)+\operatorname{arctanh}\left[\frac{\tan \theta_{0}}{\sqrt{b}}\right] .
$$




\section{Acknowledgements}

The authors are grateful for the many productive discussions with and suggestions from B. I. Cohen, L. Divol, R. K Kirkwood, and A. B. Langdon. The authors further thank the Multiprogrammatic and Institutional Computing Initiative at Lawrence Livermore National Laboratory, which provided the computer resources for simulations.

\section{References}

[1] J. D. Lindl, P. Amendt, R. L. Berger, S. G. Glendinning, S. H. Glenzer, S. W. Haan, R. L. Kauffman, O. L. Landen, L. J. Suter, The physics basis for ignition using indirect drive targets on the NIF, Phys. Plas. 11 (2) (2003) 339-491, doi:10.1063/1.1578638.

[2] W. L. Kruer, S. C. Wilks, B. B. Afeyan, R. K. Kirkwood, Energy transfer between crossing laser beams, Phys. Plas. 3 (1) (1996) 382-385.

[3] R. K. Kirkwood, B. B. Afeyan, W. L. Kruer, B. J. MacGowan, J. D. Moody, D. S. Montgomery, D. M. Pennington, T. L. Weiland, S. C. Wilks, Observation of energy transfer between frequency-matched laser beams in a large-scale plasma, Phys. Rev. Lett. 76 (12) (1996) 2065(4), doi:10.1103/PhysRevLett.76.2065.

[4] R. K. Kirkwood, B. J. MacGowan, D. S. Montgomery, B. B. Afeyan, W. L. Kruer, D. M. Pennington, S. C. Wilks, J. D. Moody, K. Wharton, C. A. Back, K. G. Estabrook, S. H. Glenzer, M. A. Blain, R. L. Berger, D. E. Hinkel, B. F. Lasinski, E. A. Williams, D. Munro, B. H. Wilde, C. Rousseaux, Observation of multiple mechanisms for stimulating ion waves in ignition scale plasmas, Phys. 
Plas. 4 (5) (1997) 1800-1810, doi:10.1063/1.872293.

[5] K. B. Wharton, R. K. Kirkwood, S. H. Glenzer, K. G. Estabrook, B. B. Afeyan, B. I. Cohen, J. D. Moody, C. Joshi, Observation of energy transfer between identical-frequency laser beams in a flowing plasma, Phys. Rev. Lett. 81 (11) (1998) 2248-2251, doi:10.1103/PhysRevLett.81.2248.

[6] K. B. Wharton, R. K. Kirkwood, S. H. Glenzer, K. G. Estabrook, B. B. Afeyan, B. I. Cohen, J. D. Moody, B. J. MacGowan, C. Joshi, Observation of resonant energy transfer between identical-frequency laser beams, Phys. Plas 6 (5) (1999) 2144-2149, doi:10.1063/1.873500.

[7] V. V. Eliseev, W. Rozmus, V. T. Tikhonchuk, C. E. Capjack, Interaction of crossed laser beams with plasmas, Phys. Plas. 3 (6) (1996) 2215-2217, doi:10.1063/1.871703.

[8] H. A. Rose, S. Ghosal, Nonlinear theory of power transfer between multiple crossed laser beams in a flowing plasma, Phys. Plasma 5 (5) (1998) 1461-1466, doi:10.1063/1.872804.

[9] C. J. McKinstrie, J. S. Li, R. E. Giacone, H. X. Vu, Two-dimensional analysis of the power transfer between crossed laser beams, Phys. Plas. 3 (7) (1996) 2686-2692, doi:10.1063/1.871721.

[10] C. J. McKinstrie, A. V. Kanaev, V. T. Tikhonchuk, R. E. Giacone, H. X. Vu, Three-dimensional analysis of the power transfer between crossed laser beams, Phys. Plas. 5 (4) (1998) 1142-1147, doi:10.1063/1.872645.

[11] C. J. McKinstrie, V. A. Smalyuk, R. E. Giacone, H. X. Vu, Power exchange between crossed laser beams and the associated frequency cascade, Phys. Rev. E 55 (2) (1997) 2044-2047, doi:10.1103/PhysRevE.55.2044.

[12] S. Skupsky, R. W. Short, T. Kessler, R. S. Craxton, S. Letzring, J. M. Soures, 
Improved laser-beam uniformity using the angular dispersion of frequencymodulated light, J. Appl. Phys. 66 (8) (1989) 3456-3462, doi:10.1063/1.344101.

[13] R. K. Kirkwood, P. E. Young, J. D. Moody, A. B. Langdon, B. I. Cohen, E. A. Williams, M. R. Dorr, J. A. Hittinger, R. Berger, L. J. Suter, L. Divol, S. H. Glenzer, O. L. Landen, W. Seka, Observation of saturation of energy transfer between co-propagating beams in a flowing plasma, Phys. Rev. Lett. 89 (21) (2002) 215003(4), doi:10.1103/PhysRevLett.89.215003.

[14] A. B. Langdon, B. F. Lasinski, Electromagnetic and relativistic plasma simulation models, in: B. Adler, S. Fernbach, M. Rotenberg, J. Killeen (Eds.), Methods in Computational Physics, Vol. 16, Academic Press, New York, 1976, pp. 327-366.

[15] B. I. Cohen, B. F. Lasinski, A. B. Langdon, E. A. Williams, K. B. Wharton, R. K. Kirkwood, K. G. Estabrook, Resonant stimulated Brillouin interaction of opposed laser beams in a drifting plasma, Phys. Plas. 5 (9) (1998) 3408-3415, doi:10.1063/1.873055.

[16] E. A. Williams, B. I. Cohen, L. Divol, M. R. Dorr, J. A. Hittinger, D. E. Hinkel, A. B. Langdon, R. K. Kirkwood, D. H. Froula, S. H. Glenzer, Effects of ion trapping on crossed-laser-beam stimulated Brillouin scattering, Phys. Plasmas 11 (1) (2004) 231-244, doi:10.1063/1.1630573.

[17] M. Dorr, F. X. Garaizar, J. A. F. Hittinger, Simulation of laser plasma filamentation using adaptive mesh refinement, J. Comput. Phys. 177 (2002) 233-263, doi:10.1006/jcph.2001.6985.

[18] M. N. Rosenbluth, Parametric instabilities in inhomogeneous media, Phys. Rev. Lett. 29 (9) (1972) 565-567, doi:10.1103/PhysRevLett.29.565.

[19] E. A. Williams, Extension of 'four-dimensional eikonal theory of linear mode conversion' to parametrically unstable media, Phys. Rev. Lett. 59 (23) (1987) 
2709, doi:10.1103/PhysRevLett.59.2709.

[20] R. P. Drake, E. A. Williams, Three-wave parametric amplification in timedependent media, with application to stimulated Brillouin scattering, Phys. Rev. Lett. 67 (18) (1991) 2477-2480, doi:10.1103/PhysRevLett.67.2477.

[21] The Mathworks, Inc., Natick, MA, MatLAB, www.mathworks.com (2002).

[22] A. C. Hindmarsh, A. G. Taylor, User documentation for IDA, a differentialalgebraic equation solver for sequential and parallel computers, Tech. Rep. UCRL-MA-136910, Lawrence Livermore National Laboratory (1999).

[23] G. D. Byrne, A. C. Hindmarsh, A polyalgorithm for the numerical solution of ordinary differential equations, ACM Trans. Math. Software 1 (1) (1975) 71-96.

[24] K. R. Jackson, R. Sacks-Davis, An alternative implementation of variable stepsize multistep formulas for stiff ODEs, ACM Trans. Math. Software 6 (3) (1980) $295-318$.

[25] K. E. Brenan, S. L. Campbell, L. R. Petzold, Numerical Solution of Initial-Value Problems in Differential-Algebraic Equations, second edition Edition, Vol. 14 of Classics in Applied Mathematics, SIAM, Philadelphia, PA, 1996.

[26] P. N. Brown, A. C. Hindmarsh, L. R. Petzold, Using Krylov methods in the solution of large-scale differential-algebraic systems, SIAM J. Sci. Comput. 15 (1994) 1467-1488. 


\section{List of Figures}

1 Basic configuration of lasers crossing in the vicinity of the sonic line $\left(M_{0}=1\right)$ of an expanding plasma.

2 Simplified geometry of the crossed pump and probe beams in an expanding plasma.

3 Geometry of the interaction region neglecting refraction and assuming symmetry.

4 Comparison of SSA and TDCM probe gains for the narrow beam case. Curves without markers were computed by the TDCM code. Curves marked by circles were computed using the SSA model.

5 Comparison of SSCM and TDCM probe gains for the narrow beam case. Curves without markers were computed by the TDCM code. Curves marked by diamonds were computed using the SSCM model.

6 Comparison of SSA and TDCM probe gains for the medium beam case. Curves without markers were computed by the TDCM code. Curves marked by circles were computed using the SSA model.

7 Comparison of SSCM and TDCM probe gains for the medium beam case. Curves without markers were computed by the TDCM code. Curves marked by diamonds were computed using the SSCM model.

8 Comparison of SSA and TDCM probe gains for the wide beam case. Curves without markers were computed by the TDCM code. Curves marked by circles were computed using the SSA model.

9 Comparison of SSCM and TDCM probe gains for the wide beam case. Curves without markers were computed by the TDCM code. Curves marked by diamonds were computed using the SSCM model.

10 Medium beam, short initial scale length case experiment with frozen background flow fixed for the first $20 \mathrm{ps}$ at $L_{\perp}(20 \mathrm{ps})$. 
11 Transverse velocities of the central ray intersection relative to the sonic line sound speed in an adiabatic expansion for increasing initial velocity scale lengths. All three beam widths follow the same curves. Final time corresponds to $50 \mathrm{ps}$ for the narrow beam, 500 ps for the medium beam, and 5000 ps for the wide beam.

12 Medium beam width, short scale length case with gain computed three ways. The solid curve is the gain directly computed from the unsteady coupled mode code. The dashed curve represents the gain computed using the light fields from the unsteady coupled mode code and the steady-state acoustic response (27). Finally, the dotted curve with circular symbols is the gain computed using the light fields from the unsteady coupled mode code together with the acoustic response taking into account the frequency mismatch (29). The frequency mismatch is approximated from the acoustic response directly computed by the unsteady coupled mode code using (65).

13 Medium beam case with downward shifted beams. 


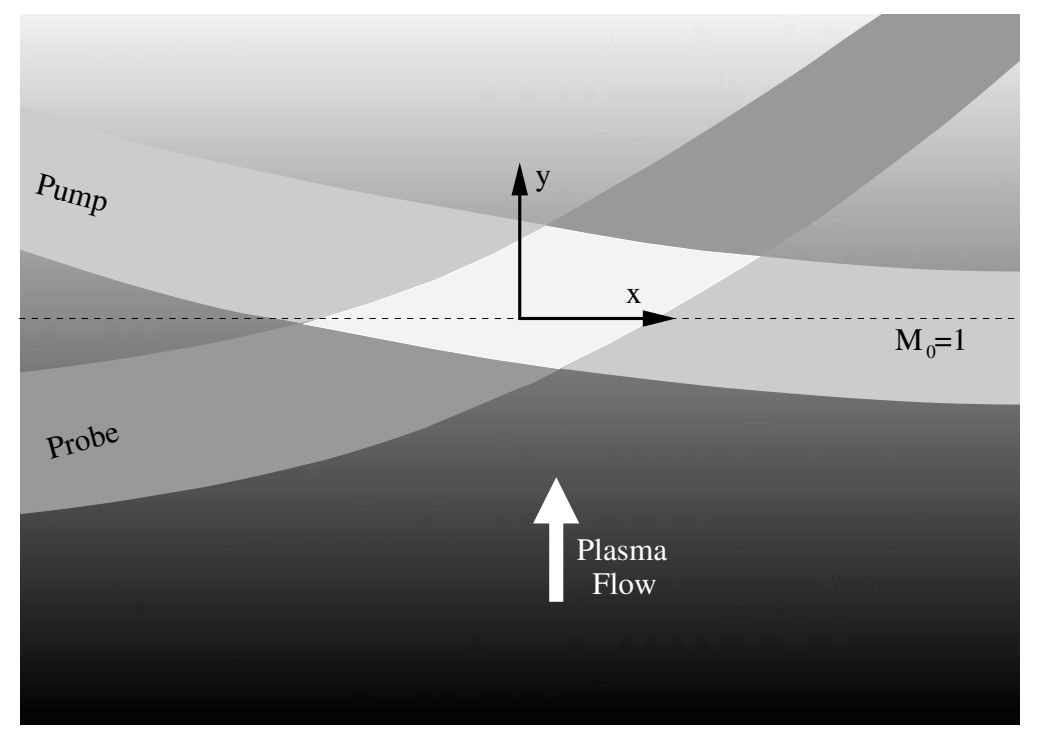

Fig. 1. Basic configuration of lasers crossing in the vicinity of the sonic line $\left(M_{0}=1\right)$ of an expanding plasma. 


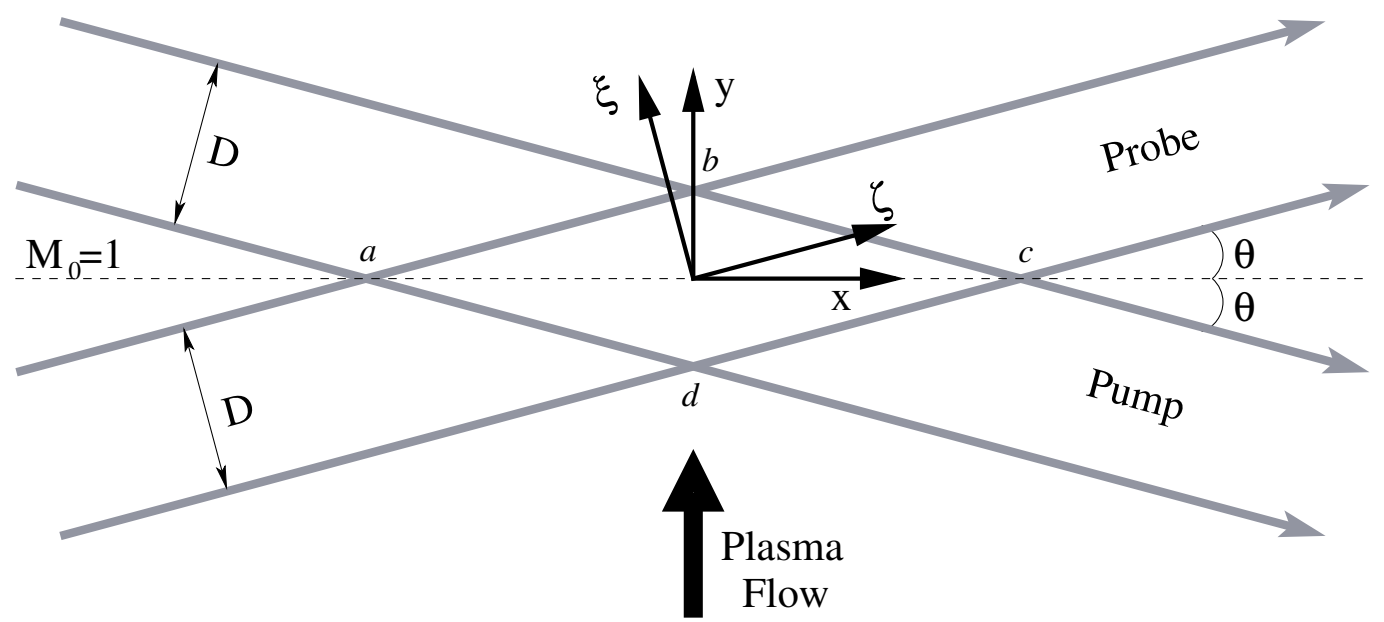

Fig. 2. Simplified geometry of the crossed pump and probe beams in an expanding plasma. 


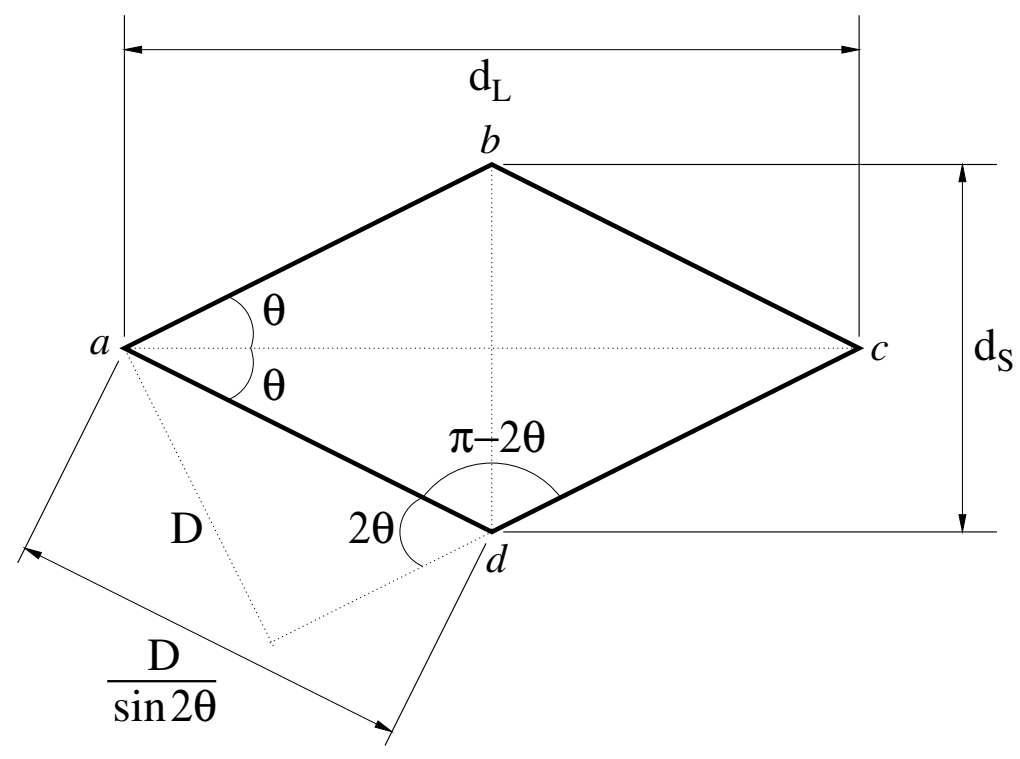

Fig. 3. Geometry of the interaction region neglecting refraction and assuming symmetry. 


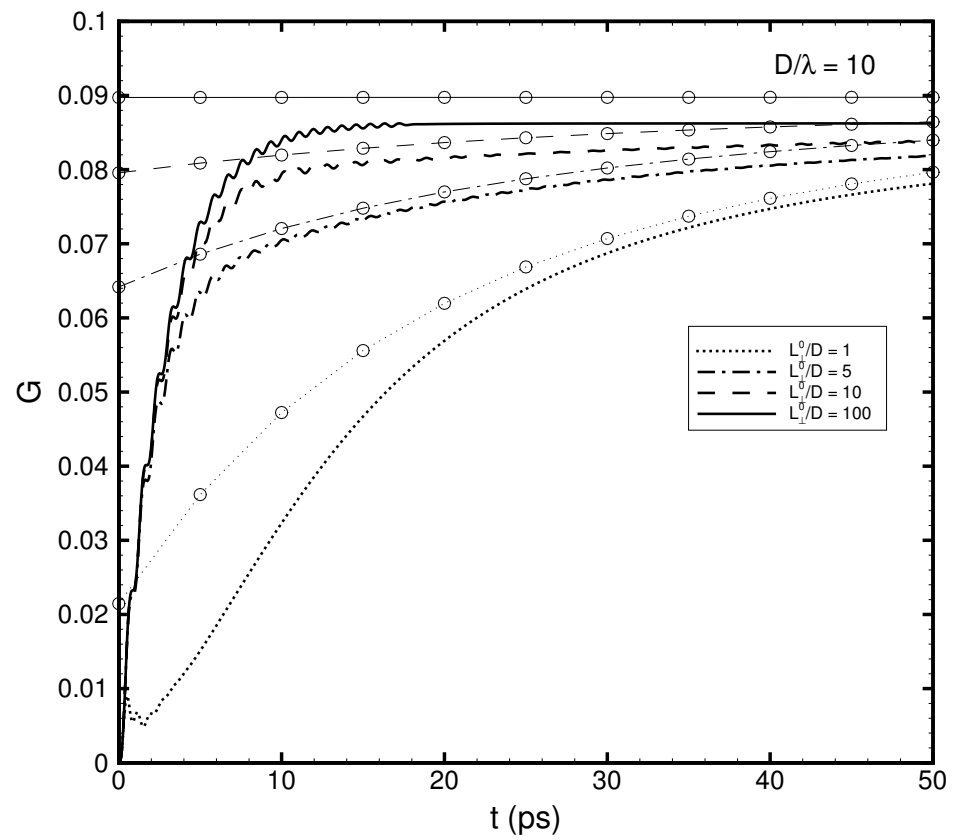

Fig. 4. Comparison of SSA and TDCM probe gains for the narrow beam case. Curves without markers were computed by the TDCM code. Curves marked by circles were computed using the SSA model. 


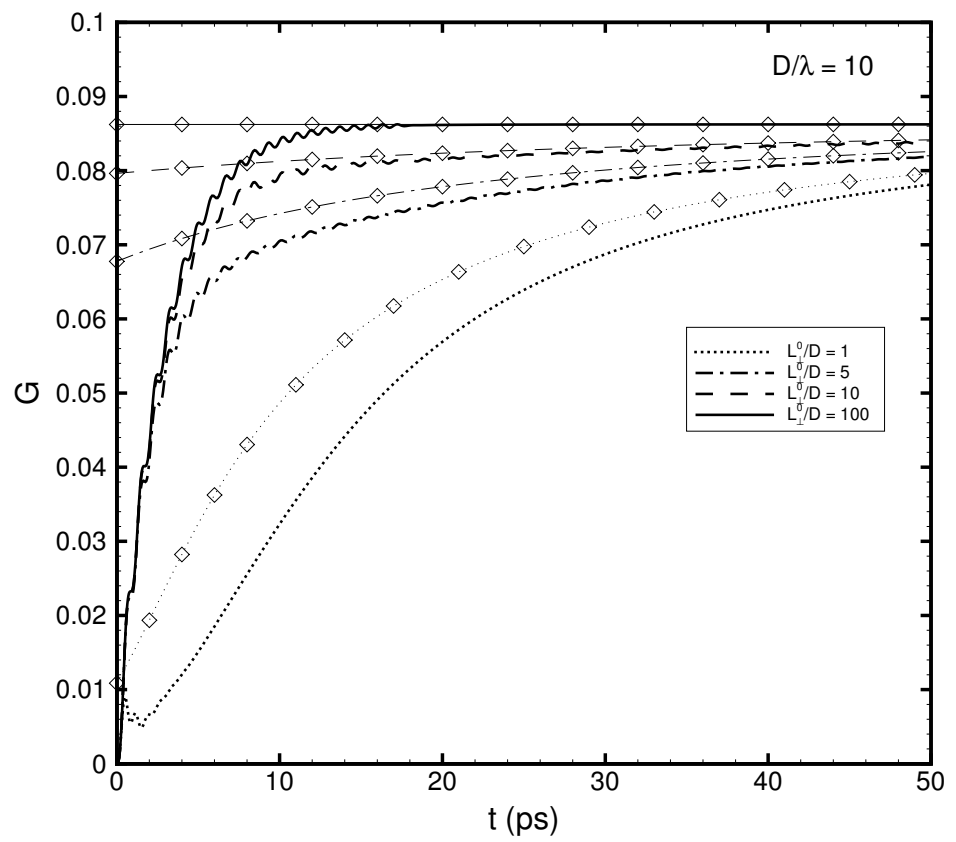

Fig. 5. Comparison of SSCM and TDCM probe gains for the narrow beam case. Curves without markers were computed by the TDCM code. Curves marked by diamonds were computed using the SSCM model. 


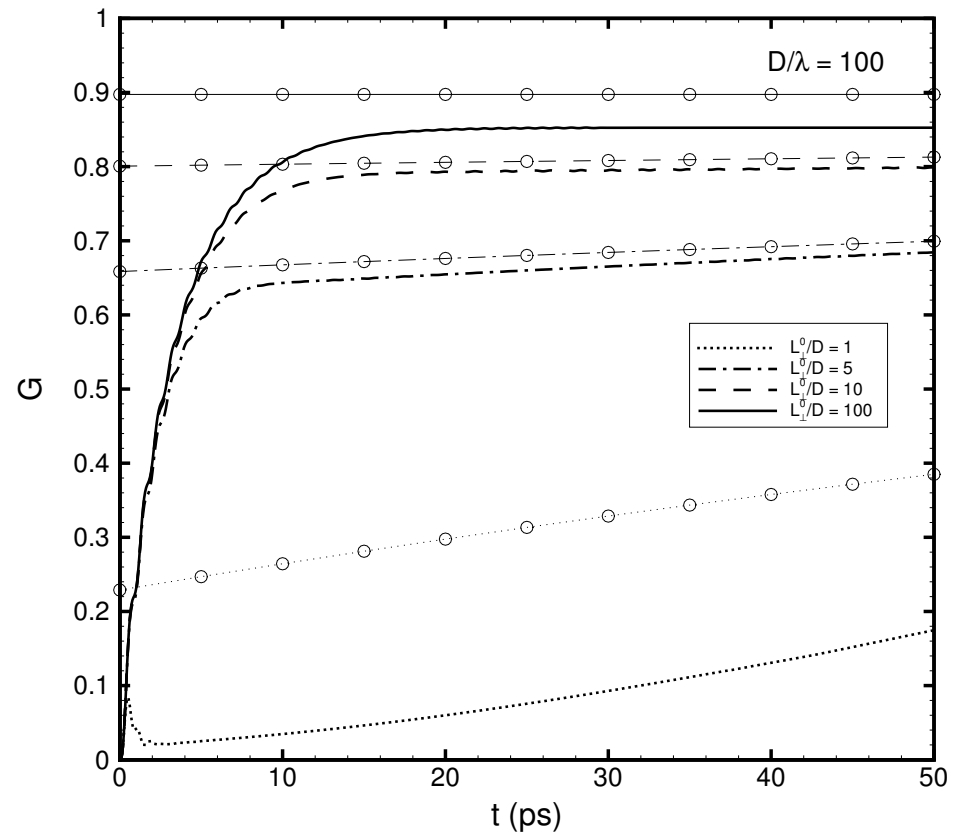

Fig. 6. Comparison of SSA and TDCM probe gains for the medium beam case. Curves without markers were computed by the TDCM code. Curves marked by circles were computed using the SSA model. 


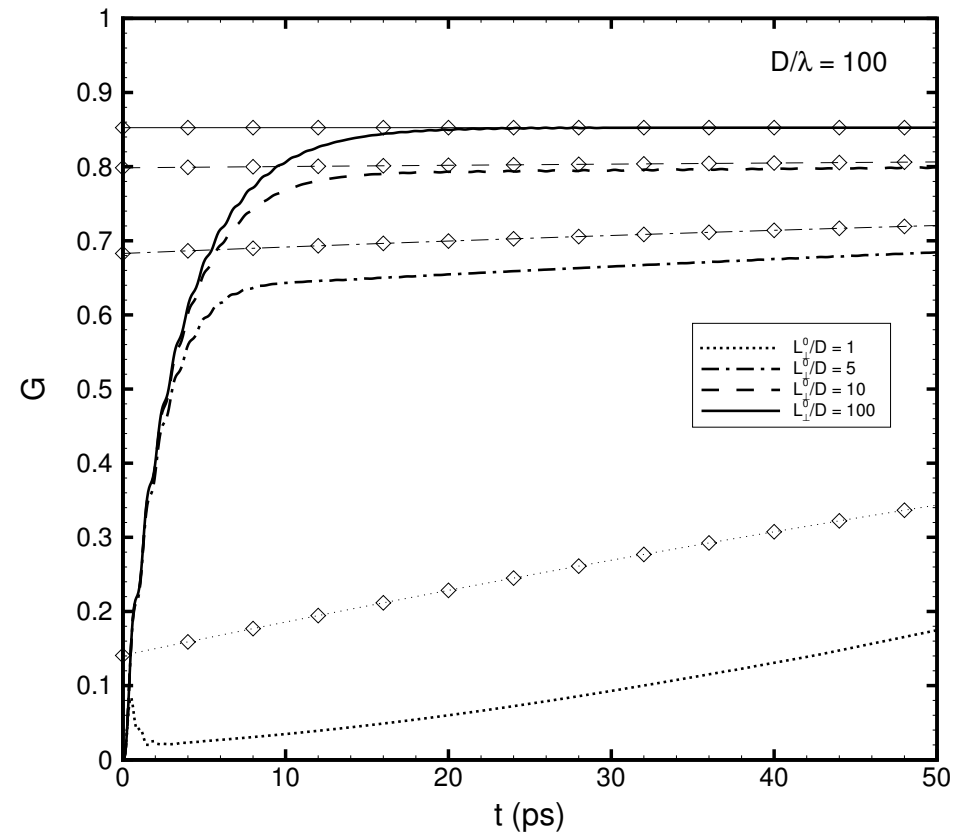

Fig. 7. Comparison of SSCM and TDCM probe gains for the medium beam case. Curves without markers were computed by the TDCM code. Curves marked by diamonds were computed using the SSCM model. 


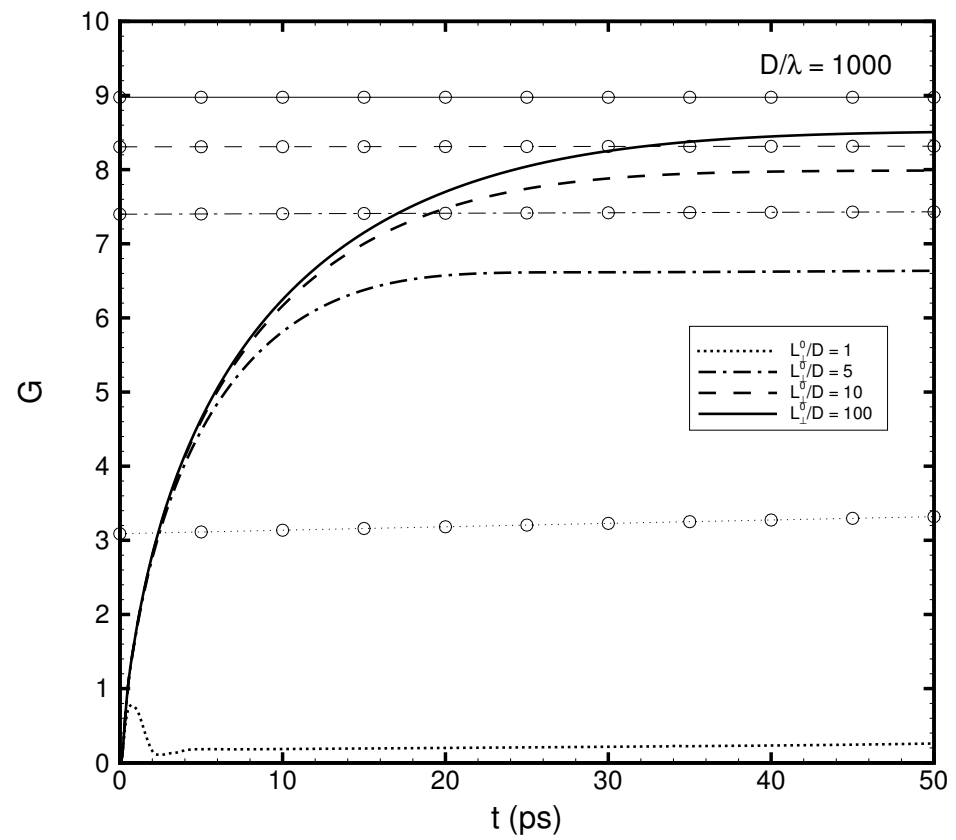

Fig. 8. Comparison of SSA and TDCM probe gains for the wide beam case. Curves without markers were computed by the TDCM code. Curves marked by circles were computed using the SSA model. 


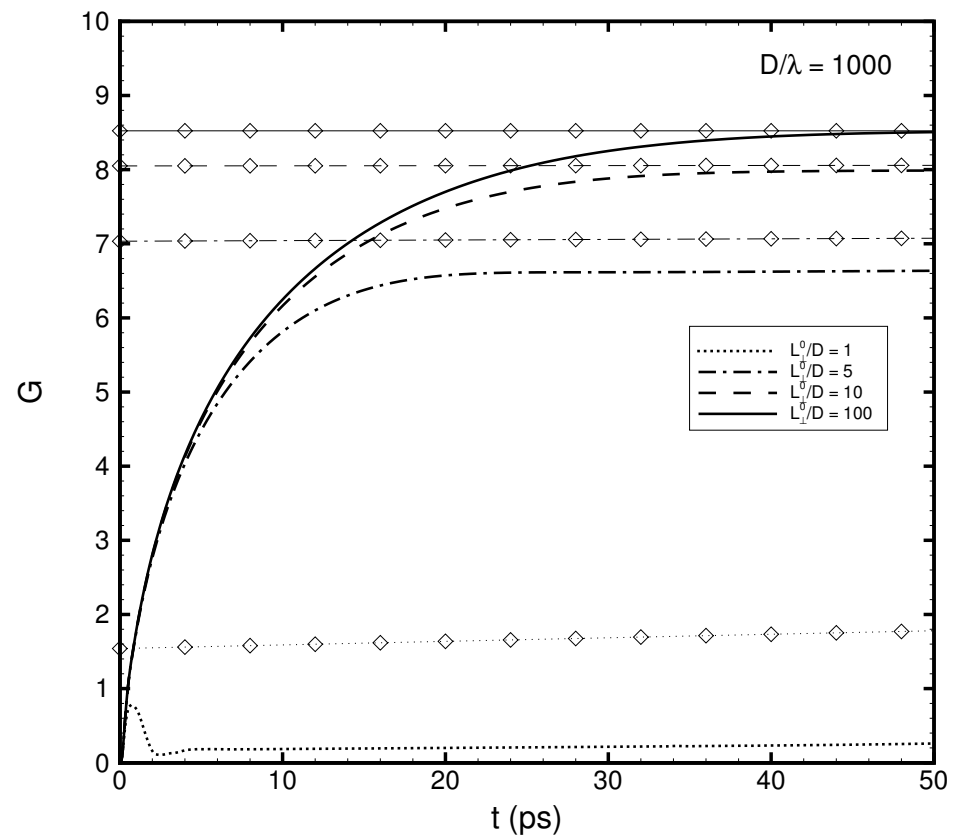

Fig. 9. Comparison of SSCM and TDCM probe gains for the wide beam case. Curves without markers were computed by the TDCM code. Curves marked by diamonds were computed using the SSCM model. 


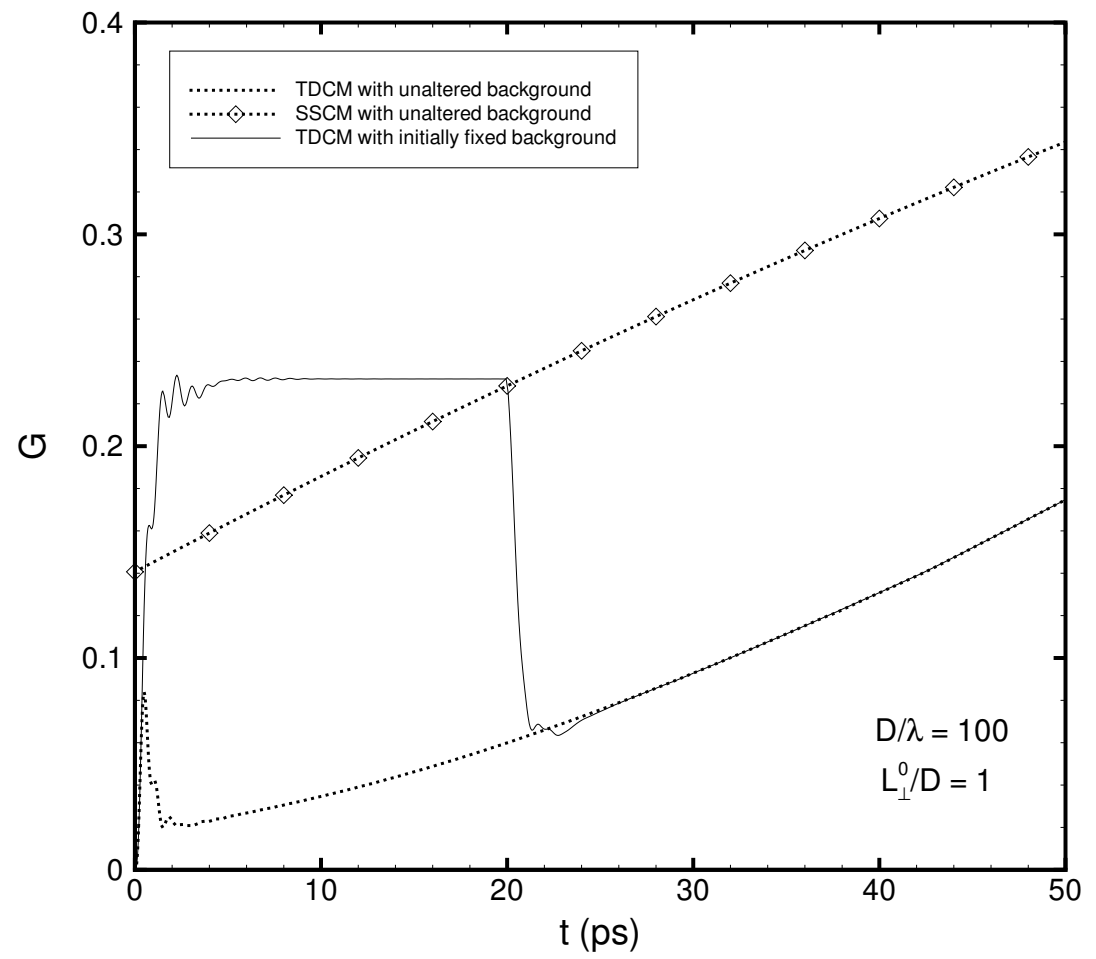

Fig. 10. Medium beam, short initial scale length case experiment with frozen background flow fixed for the first $20 \mathrm{ps}$ at $L_{\perp}(20 \mathrm{ps})$. 


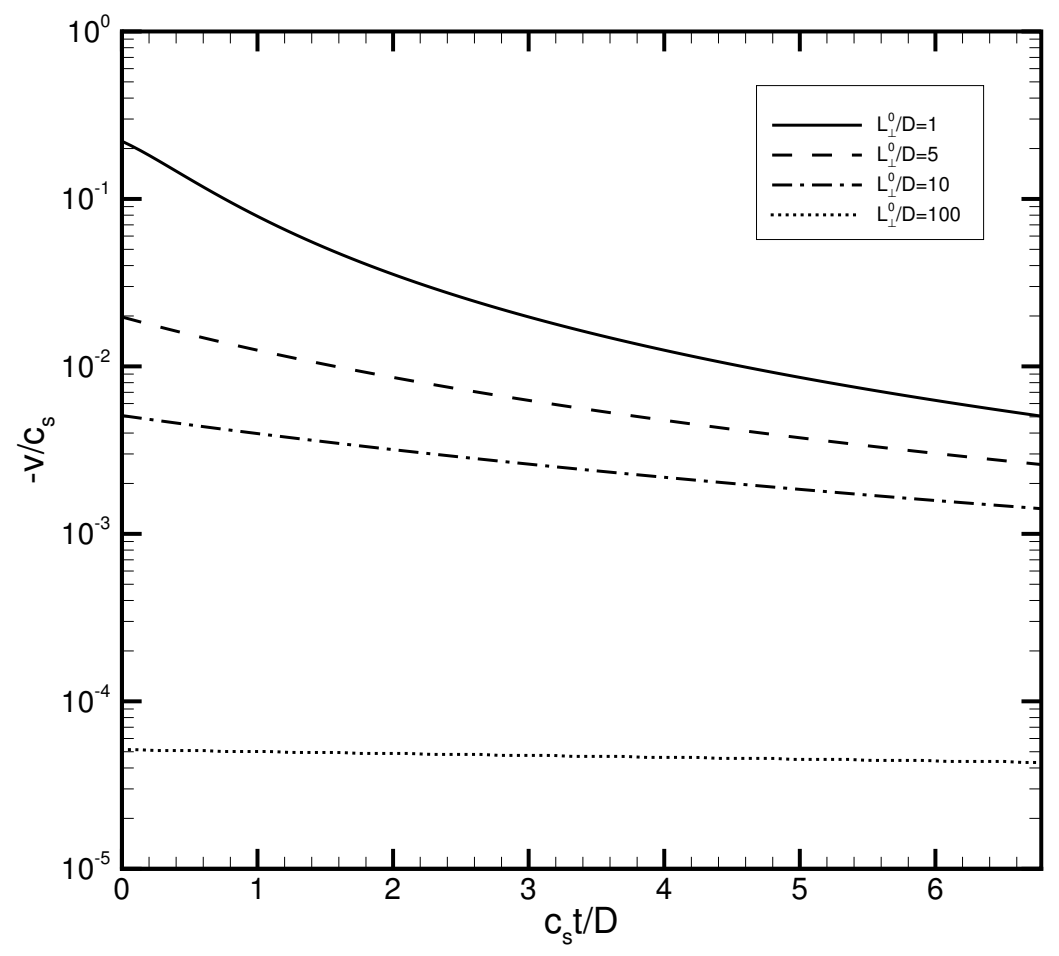

Fig. 11. Transverse velocities of the central ray intersection relative to the sonic line sound speed in an adiabatic expansion for increasing initial velocity scale lengths. All three beam widths follow the same curves. Final time corresponds to 50 ps for the narrow beam, 500 ps for the medium beam, and $5000 \mathrm{ps}$ for the wide beam. 


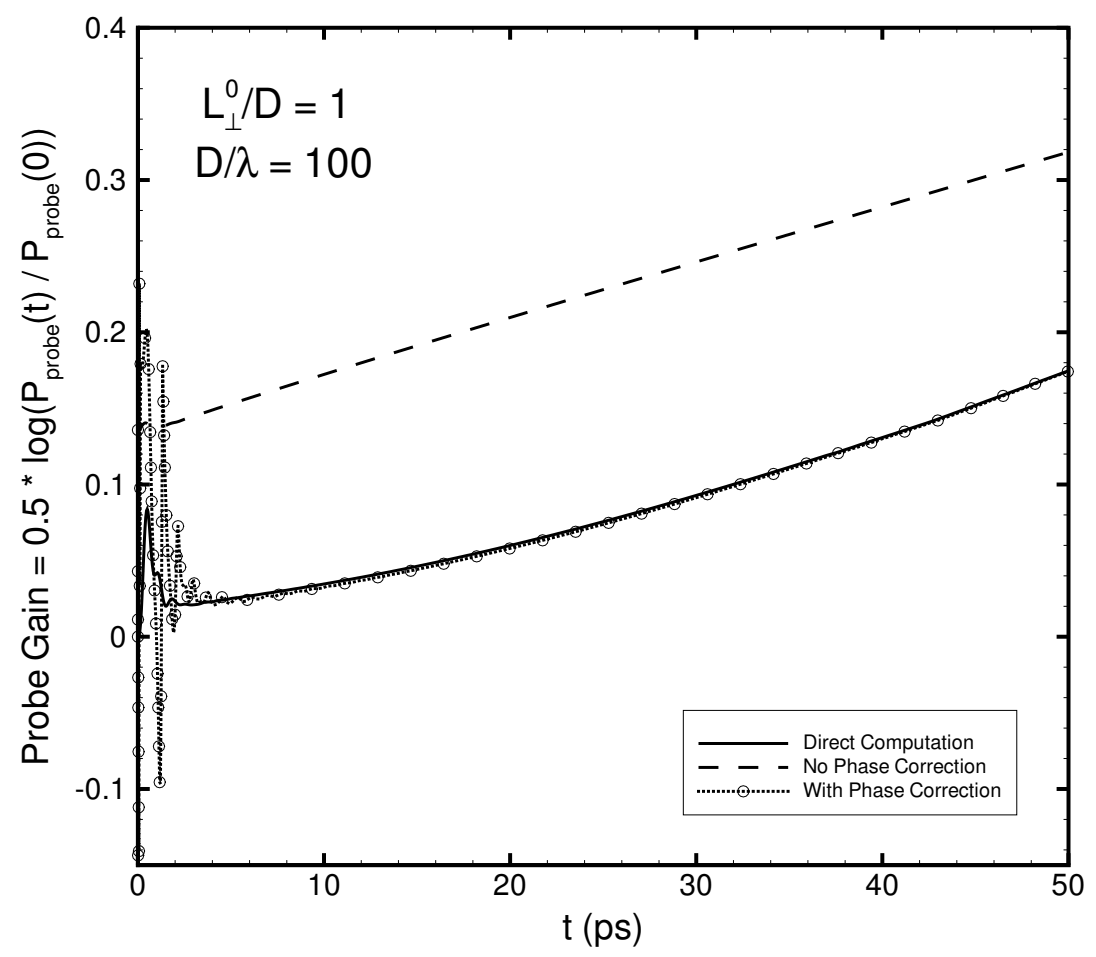

Fig. 12. Medium beam width, short scale length case with gain computed three ways. The solid curve is the gain directly computed from the unsteady coupled mode code. The dashed curve represents the gain computed using the light fields from the unsteady coupled mode code and the steady-state acoustic response (27). Finally, the dotted curve with circular symbols is the gain computed using the light fields from the unsteady coupled mode code together with the acoustic response taking into account the frequency mismatch (29). The frequency mismatch is approximated from the acoustic response directly computed by the unsteady coupled mode code using (65). 


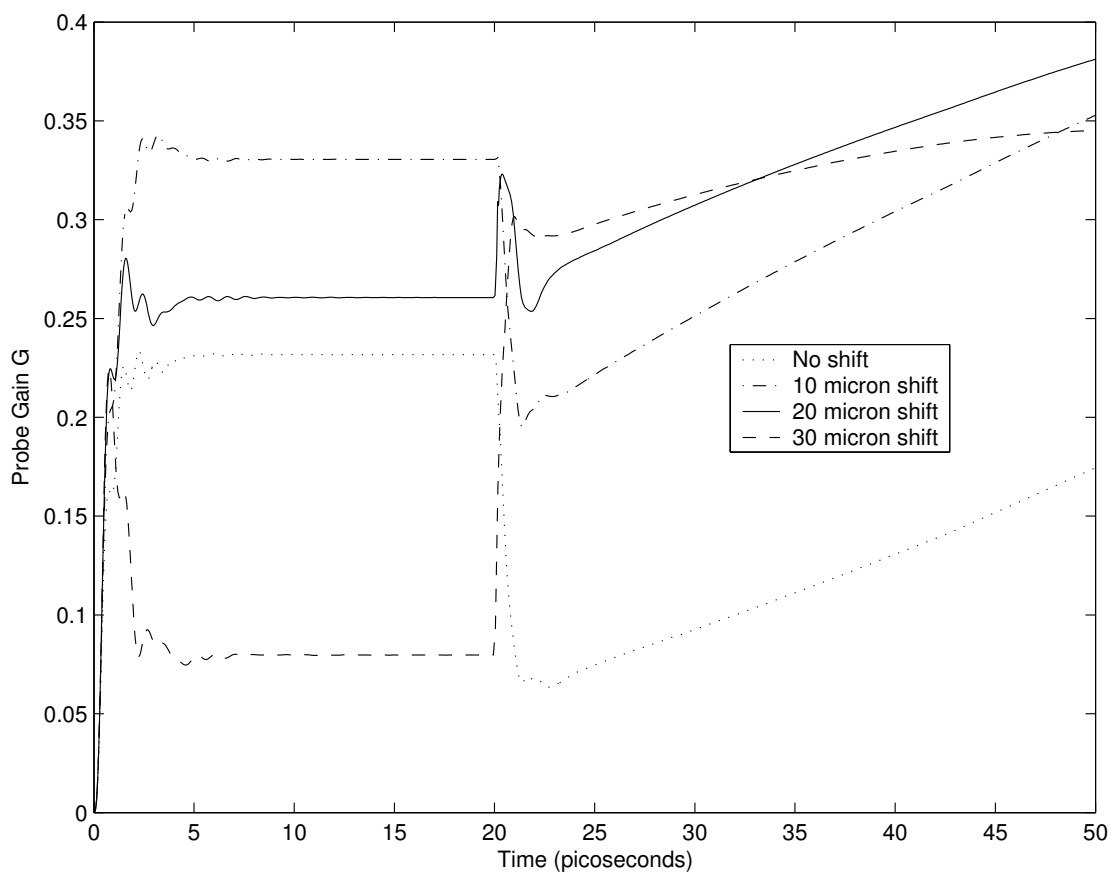

Fig. 13. Medium beam case with downward shifted beams. 


\section{List of Tables}

1 Vertices of the interaction region in the flow and probe beam coordinates. 
Table 1

\begin{tabular}{ccc} 
Vertex & $(x, y)$ & $(\zeta, \xi)$ \\
\hline$a$ & $\left(-\frac{1}{2} D \csc \theta, 0\right)$ & $\left(-\frac{1}{2} D \cot \theta, \frac{1}{2} D\right)$ \\
$b$ & $\left(0, \frac{1}{2} D \sec \theta\right)$ & $\left(\frac{1}{2} D \tan \theta, \frac{1}{2} D\right)$ \\
$c$ & $\left(\frac{1}{2} D \csc \theta, 0\right)$ & $\left(\frac{1}{2} D \cot \theta,-\frac{1}{2} D\right)$ \\
$d$ & $\left(0,-\frac{1}{2} D \sec \theta\right)$ & $\left(-\frac{1}{2} D \tan \theta,-\frac{1}{2} D\right)$
\end{tabular}

Vertices of the interaction region in the flow and probe beam coordinates. 